\title{
Lie algebroid structures and Lagrangian systems on affine bundles
}

\author{
Eduardo Martínez ${ }^{\dagger}$, Tom Mestdag ${ }^{\ddagger}$ and Willy Sarlet ${ }^{\ddagger}$ \\ $\dagger$ †epartamento de Matemática Aplicada \\ Universidad de Zaragoza, María de Luna 3, 50015 Zaragoza, Spain \\ $\ddagger$ Department of Mathematical Physics and Astronomy \\ Ghent University, Krijgslaan 281, B-9000 Ghent, Belgium
}

\begin{abstract}
As a continuation of previous papers, we study the concept of a Lie algebroid structure on an affine bundle by means of the canonical immersion of the affine bundle into its bidual. We pay particular attention to the prolongation and various lifting procedures, and to the geometrical construction of Lagrangian-type dynamics on an affine Lie algebroid.
\end{abstract}

\section{Introduction}

Since the book of Mackenzie [12], the mathematics of Lie algebroids (and groupoids) has been studied by many authors; for a non-exhaustive list of references, see for example 3, 5, 6, 9, 10, 13, 18]. The potential relevance of Lie algebroids for applications in physics and other fields of applied mathematics has gradually become more evident. In particular, contributions by Libermann 11] and Weinstein [21] have revealed the role Lie algebroids play in modelling certain problems in mechanics. The concept of 'Lagrangian equations' on Lie algebroids certainly defines an interesting generalisation of Lagrangian systems as known from classical mechanics, if only because of the more general class of differential equations it involves while preserving a great deal of the very rich geometrical structure of Lagrangian (and Hamiltonian) mechanics.

One of us [16], in particular, has produced evidence of this rich structure by showing that one can prolong a Lie algebroid in such a way that the newly obtained space has all the features of tangent bundle geometry, which are important for the geometrical construction of Lagrangian systems. That is to say, the prolonged Lie algebroid carries a Liouville-type section and a vertical endomorphism which enables the definition of a Poincaré-Cartan type 1-form, associated to a function $L$; the available exterior derivative then does the rest 
for arriving at an analogue of the symplectic structure from which Lagrangian equations can be derived.

In [20], we have started an investigation on the possible generalisation of the concept of Lie algebroids to affine bundles. Our principle motivation was to create a geometrical model which would be a natural environment for a timedependent version of Lagrange equations on Lie algebroids, as discussed for example in [21] and [16]. Since classical time-dependent mechanics is usually described on the first-jet bundle $J^{1} M$ of a manifold $M$ fibred over $\mathbb{R}$ (see e.g. [4, 14), and $J^{1} M \rightarrow M$ is an affine bundle, it looks natural to build up a timedependent generalisation in such a way that $J^{1} M \rightarrow M$ is the image bundle of the anchor map of a Lie algebroid structure on some affine bundle $E \rightarrow M$. An additional indication that such a set-up is well suited came from a naive calculus of variations approach, which gives a clue on the analytical format one should expect for such time-dependent Lagrange equations (see [19]).

Lie algebroids on vector bundles are known to give rise, among other things, to a linear Poisson structure on the dual bundle, as well as a coboundary operator on its Grassmann algebra; in fact, these properties equivalently characterise the Lie algebroid structure. One of the features of the approach to affine algebroids adopted in 20] was our specific choice to develop, in a direct way, a consistent theory of forms on sections of an affine bundle and their exterior calculus. By contrast, however, in the context of briefly mentioning the related Poisson structure in the concluding remarks, we did announce a forthcoming different approach, which would be based on the fact that an affine bundle can be regarded as an affine sub-bundle of a vector bundle, namely the dual of its extended dual. This is the line of reasoning we will develop here; it could be termed 'indirect' because it makes use of an imbedding into a larger bundle, but it has some marked advantages, such as the fact that proving a number of properties becomes much easier and that new insights come to the forefront. As a matter of fact, one readily recognises via this approach that much (if not all) of the theory of affine Lie algebroids can be developed without needing an extra fibration of the base manifold over $\mathbb{R}$. We will accordingly start our present analysis in this more general set-up and briefly come back to the special case appropriate for time-dependent systems in the concluding remarks.

Note: while the very last editing of this paper was being done, we have been informed of similar investigations on affine algebroids, which have been carried out by Grabowski et al [7]. The reader may find it instructive to compare the two simultaneous developments, which are related to each other up to and including our Section 6.

The scheme of the paper is as follows. A fairly detailed description of purely algebraic aspects of the theory is given in Sections 2 to 4; it involves the introduction of the concept of a Lie algebra over an affine space and aspects of exterior calculus. A Lie algebroid structure on a general affine bundle $\tau: E \rightarrow M$ is defined in Section 5: essentially, it comes from a classical Lie algebroid on the dual $\tilde{E}$ of the extended dual $E^{\dagger}$ of $E$, with the property that the bracket of sections in the image of the inclusion $i: E \rightarrow \tilde{E}$, lies in the image of the underlying vector bundle. Equivalent characterisations of this property can be found 
in the subsequent section on the exterior differential and the associated Poisson structure. Section 7 presents a number of simple examples of affine algebroids. The important concept of prolongation of an algebroid is discussed in Section 8: starting from a general construction on vector algebroids, it is shown that the prolonged bundle inherits the affine structure coming from $E$ when the vector algebroid is the one on $\tilde{E}$. For the specific case of interest, it is further shown (see Section 9) that there is a canonical map which gives rise to a 'vertical endomorphism' on sections of the prolonged bundle. Natural constructions which are then available are complete and vertical lifts ; they play a role in the geometric definition of Lagrangian systems on affine Lie algebroids, presented in Section 10.

\section{Immersion of an affine space in a vector space}

Let $A$ be an affine space modelled on a vector space $V$, and let $A^{\dagger}=\operatorname{Aff}(A, \mathbb{R})$ be the extended dual of $A$, that is, the vector space of all affine maps from $A$ to the real line. We consider the bidual $\tilde{A}$ of $A$, in the sense $\tilde{A}=\left(A^{\dagger}\right)^{*}$. It is well known that in the case of a vector space $V$, the bidual $\tilde{V}=\left(V^{*}\right)^{*}$ is isomorphic to $V$. In the case of an affine space, the bidual includes 'a copy' of $A$, as it is shown in the following statement.

Proposition 1. The map $i: A \rightarrow \tilde{A}$ given by $i(a)(\varphi)=\varphi(a)$ is an injective affine map, whose associated vector map is $\boldsymbol{i}: V \rightarrow \tilde{A}$ given by $\boldsymbol{i}(\boldsymbol{v})(\varphi)=\boldsymbol{\varphi}(\boldsymbol{v})$

Proof. If $a \in A$ and $\boldsymbol{v} \in V$, then for all $\varphi \in A^{\dagger}$,

$$
i(a+\boldsymbol{v})(\varphi)=\varphi(a+\boldsymbol{v})=\varphi(a)+\varphi(\boldsymbol{v})=i(a)(\varphi)+\boldsymbol{i}(\boldsymbol{v}),
$$

from which it follows that $i$ is an affine map whose associated linear map is $\boldsymbol{i}$.

To prove that $i$ is injective, it suffices to prove that $i$ is injective, which is obvious since if $\boldsymbol{v}$ is an element in the kernel of $\boldsymbol{i}$ then $\boldsymbol{i}(\boldsymbol{v})(\boldsymbol{\varphi})=\boldsymbol{\varphi}(\boldsymbol{v})=0$ for all $\varphi \in V^{*}$, hence $\boldsymbol{v}=0$.

The vector space $\tilde{A}$ is foliated by hyperplanes parallel to the image of $\boldsymbol{i}$. Every vector $z \in \tilde{A}$ is either of the form $z=\boldsymbol{i}(\boldsymbol{v})$ for some $\boldsymbol{v} \in V$ or of the form $z=\lambda i(a)$ for some $\lambda \in \mathbb{R} \backslash\{0\}$ and $a \in A$. Moreover, $\lambda, a$ and $\boldsymbol{v}$ are uniquely determined by $z$. The image of the map $i$ consists of the points for which $\lambda=1$. To understand this description in more detail, we will prove that we have an exact sequence of vector spaces $0 \longrightarrow V \stackrel{i}{\longrightarrow} \tilde{A} \longrightarrow \mathbb{R} \longrightarrow 0$. To this end we consider the dual sequence.

Proposition 2. Let $l: \mathbb{R} \rightarrow A^{\dagger}$ be the map that associates to $\lambda \in \mathbb{R}$ the constant function $\lambda$ on $A$. Let $k: A^{\dagger} \rightarrow V^{*}$ the map that associates to every affine function on $A$ the corresponding linear function on $V$. Then, the sequence of vector spaces

$$
0 \longrightarrow \mathbb{R} \stackrel{l}{\longrightarrow} A^{\dagger} \stackrel{k}{\longrightarrow} V^{*} \longrightarrow 0
$$

is exact. 
Proof. Indeed, it is clear that $l$ is injective, $k$ is surjective and $k \circ l=0$, so that $\operatorname{Im}(l) \subset \operatorname{Ker}(k)$. If $\varphi \in A^{\dagger}$ is in the kernel of $k$, that is the linear part of $\varphi$ vanishes, then for every pair of points $a$ and $b=a+\boldsymbol{v}$ we have that

$$
\varphi(b)=\varphi(a+\boldsymbol{v})=\varphi(a)+\varphi(\boldsymbol{v})=\varphi(a),
$$

that is $\varphi$ is constant, and hence in the image of $l$.

The dual map of $k$ is $\boldsymbol{i}$, since for $\boldsymbol{v} \in V$ we have

$$
\langle k(\varphi), \boldsymbol{v}\rangle=\langle\boldsymbol{\varphi}, \boldsymbol{v}\rangle=\langle\varphi, \boldsymbol{i}(\boldsymbol{v})\rangle
$$

The dual map $j$ of the map $l$ is given by $j(\alpha i(a)+i \boldsymbol{v})=\alpha$. Indeed, for every $\lambda \in \mathbb{R}$ we have

$$
j(z) \lambda=\langle z, l(\lambda)\rangle=\langle\alpha i(a)+\boldsymbol{i}(\boldsymbol{v}), l(\lambda)\rangle=\alpha\langle i(a)), l(\lambda)\rangle+\langle i(\boldsymbol{v}), l(\lambda)\rangle=\alpha \lambda
$$

It follows that

Corollary 1. If $A$ is finite dimensional, then the sequence

$$
0 \longrightarrow V \stackrel{i}{\longrightarrow} \tilde{A} \stackrel{j}{\longrightarrow} \mathbb{R} \longrightarrow 0
$$

is exact.

Note that in this way we can clearly identify the image of $V$ as the hyperplane of $\tilde{A}$ with equation $j(z)=0$, and the image of $A$ as the hyperplane of $\tilde{A}$ with equation $j(z)=1$, in other words

$$
\boldsymbol{i}(V)=j^{-1}(0) \quad \text { and } \quad i(V)=j^{-1}(1) .
$$

Note in passing that if we have an exact sequence $0 \longrightarrow V \stackrel{\alpha}{\longrightarrow} W \stackrel{j}{\longrightarrow} \mathbb{R} \longrightarrow 0$, then we can define $A=j^{-1}(1)$; it follows that $A$ is an affine space modelled on the vector space $V$ and $W$ is canonically isomorphic to $\tilde{A}$. The isomorphism is the dual map of $\Psi: W^{*} \rightarrow A^{\dagger}, \Psi(\phi)(a)=\phi(i(a))$, where $i: A \rightarrow W$ is the canonical inclusion.

We now discuss the construction of a basis for $A^{\dagger}$. Let $\left(O,\left\{\boldsymbol{e}_{i}\right\}\right)$ be an affine frame on $A$. Thus every point $a$ has a representation $a=O+v^{i} \boldsymbol{e}_{i}$. The family of affine maps $\left\{e^{0}, e^{1}, \ldots, e^{n}\right\}$ given by

$$
e^{0}(a)=1 \quad e^{i}(a)=v^{i},
$$

is a basis for $A^{\dagger}$. If $\varphi \in A^{\dagger}$, and we put $\varphi_{0}=\varphi(O)$ and $\varphi_{i}=\varphi\left(\boldsymbol{e}_{i}\right)$, then $\varphi=\varphi_{0} e^{0}+\varphi_{i} e^{i}$.

It is to be noticed that, contrary to $e^{1}, \ldots, e^{n}$, the map $e^{0}$ does not depend on the frame we have chosen for $A$. In fact, $e^{0}$ coincides with the map $j$.

Let now $\left\{e_{0}, e_{1}, \ldots, e_{n}\right\}$ denote the basis of $\tilde{A}$ dual to $\left\{e^{0}, e^{1}, \ldots, e^{n}\right\}$. Then the image of the canonical immersion is given by

$$
i(O)=e_{0} \quad \boldsymbol{i}\left(\boldsymbol{e}_{i}\right)=e_{i}
$$


from which it follows that for $a=O+v^{i} \boldsymbol{e}_{i}$, we have $i(a)=e_{0}+v^{i} e_{i}$. If we denote by $\left(x^{0}, x^{1}, \ldots, x^{n}\right)$ the coordinate system on $\tilde{A}$ associated to the basis $\left\{e_{0}, \ldots, e_{n}\right\}$, then the equation of the image of the map $i$ is $x^{0}=1$, while the equation of the image of $i$ is $x^{0}=0$.

Coordinates in $\tilde{A}^{*}=A^{\dagger}$ associated to the basis above will be denoted by $\left(\mu_{0}, \mu_{1}, \ldots, \mu_{n}\right)$, that is $\mu_{\alpha}(\varphi)=\left\langle\boldsymbol{e}_{\alpha}, \varphi\right\rangle$ for every $\varphi \in A^{\dagger}$.

\section{$3 \quad$ Lie algebra structure over an affine space}

Implicit in our previous paper 20] is the following definition of a Lie algebra over an affine space.

Definition 1. Let $A$ be an affine space over a vector space $V$. A Lie algebra structure on $A$ is given by

- a Lie algebra structure [, ] on $V$, and

- an action by derivations of $A$ on $V$, i.e. a map $D: A \times V \rightarrow V,(a, v) \mapsto$ $D_{a} \boldsymbol{v}$ with the properties

$$
\begin{aligned}
& D_{a}(\lambda \boldsymbol{v})=\lambda D_{a} \boldsymbol{v} \\
& D_{a}(\boldsymbol{v}+\boldsymbol{w})=D_{a} \boldsymbol{v}+D_{a} \boldsymbol{w} \\
& D_{a}[\boldsymbol{v}, \boldsymbol{w}]=\left[D_{a} \boldsymbol{v}, \boldsymbol{w}\right]+\left[\boldsymbol{v}, D_{a} \boldsymbol{w}\right]
\end{aligned}
$$

- satisfying the compatibility property

$$
D_{a+\boldsymbol{v}} \boldsymbol{w}=D_{a} \boldsymbol{w}+[\boldsymbol{v}, \boldsymbol{w}] .
$$

Incidentally, it is sufficient to require in the first item that the bracket on $V$ is $\mathbb{R}$-bilinear and skew-symmetric, since the Jacobi identity then follows from the requirements on $D_{a}$.

If we use a bracket notation $[a, \boldsymbol{v}] \equiv D_{a} \boldsymbol{v}$, then the conditions in the definition above read

$$
\begin{aligned}
& {[a, \lambda \boldsymbol{v}]=\lambda[a, \boldsymbol{v}]} \\
& {[a, \boldsymbol{v}+\boldsymbol{w}]=[a, \boldsymbol{v}]+[a, \boldsymbol{w}]} \\
& {[a,[\boldsymbol{v}, \boldsymbol{w}]]=[[a, \boldsymbol{v}], \boldsymbol{w}]+[\boldsymbol{v},[a, \boldsymbol{w}]]} \\
& {[a+\boldsymbol{v}, \boldsymbol{w}]=[a, \boldsymbol{w}]+[\boldsymbol{v}, \boldsymbol{w}] .}
\end{aligned}
$$

This allows us to define a bracket of elements of $A$ by putting

$$
[\boldsymbol{v}, a]=-[a, \boldsymbol{v}]
$$

and, if $b=a+\boldsymbol{v}$, then

$$
[a, b]=[a, \boldsymbol{v}] .
$$

This bracket is skew-symmetric by construction and also satisfies a Jacobi-type property. 
Theorem 1. A Lie algebra structure over an affine space $A$ is equivalent to a Lie algebra extension of the trivial Lie algebra $\mathbb{R}$ by $V$. Explicitly, it is equivalent to the exact sequence of vector spaces $0 \longrightarrow V \stackrel{i}{\longrightarrow} \tilde{A} \stackrel{j}{\longrightarrow} \mathbb{R} \longrightarrow 0$ being an exact sequence of Lie algebras.

Proof. If the exact sequence is one of Lie algebras, we of course have a Lie algebra structure on $V$ and the map $D$ determined by $D_{a} \boldsymbol{v}=[i(a), \boldsymbol{i}(\boldsymbol{v})]$ satisfies all requirements to define a Lie algebra structure on $A$.

Conversely, assume we have a Lie algebra structure on the affine space $A$. If we fix an element $a \in A$, then every element $z \in \tilde{A}$ can be written in the form $z=\lambda i(a)+\boldsymbol{i}(\boldsymbol{v})$. We can define a bracket of two elements $z_{1}=\lambda_{1} i(a)+\boldsymbol{i}\left(\boldsymbol{v}_{1}\right)$ and $z_{2}=\lambda_{2} i(a)+\boldsymbol{i}\left(\boldsymbol{v}_{2}\right)$ by

$$
\left[z_{1}, z_{2}\right]=\boldsymbol{i}\left(\left[\boldsymbol{v}_{1}, \boldsymbol{v}_{2}\right]+\lambda_{1} D_{a} \boldsymbol{v}_{2}-\lambda_{2} D_{a} \boldsymbol{v}_{1}\right)
$$

This bracket is clearly bi-linear and skew-symmetric, and a straightforward calculation shows that it satisfies the Jacobi identity. Moreover, the definition does not depend on the choice of the point $a$; if $a^{\prime}$ is another point in $A$, then $a^{\prime}=a+\boldsymbol{w}$ for some $\boldsymbol{w} \in V$ and the compatibility condition implies that the result is independent of that choice. Finally, it is obvious that the maps $i$ and $j$ then are Lie algebra homomorphisms.

Notice that the only condition for a Lie algebra structure on $\tilde{A}$ to be an extension of $\mathbb{R}$ by $V$ is that the bracket takes values in $V$, symbolically: $[\tilde{A}, \tilde{A}] \subset$ $V$.

Once we have chosen an affine frame on $A$, we have that the bracket on $\tilde{A}$ is determined by the brackets of the associated basis elements. These must be of the form

$$
\left[e_{0}, e_{0}\right]=0 \quad\left[e_{0}, e_{j}\right]=C_{0 j}^{k} e_{k} \quad\left[e_{i}, e_{j}\right]=C_{i j}^{k} e_{k},
$$

since all brackets must take values in the image of the map $\boldsymbol{i}$.

It is well known that a Lie algebra structure on a vector space defines, and is defined by, a linear Poisson structure on the dual vector space. In the light of the results of the previous section we have a Poisson bracket on $V^{*}$ and one on $A^{\dagger}$. Furthermore, $\tilde{A}$ being an extension of $\mathbb{R}$ by $V$, we have that the Poisson structure $\Lambda_{A^{\dagger}}$ is an extension by $\Lambda_{V^{*}}$ of $\Lambda_{\mathbb{R}}=0$ (see [2] for the details on Poisson extensions and their relations to Lie algebra extensions). Therefore, once we have fixed a point $a \in A$, we have a splitting of the sequence $0 \longrightarrow V \stackrel{i}{\longrightarrow} \tilde{A} \stackrel{j}{\longrightarrow} \mathbb{R} \longrightarrow 0$ given by $h(\lambda)=\lambda i(a)$, and the Poisson tensor can be written as $\Lambda_{A^{\dagger}}=\Lambda_{V^{*}}+X_{a} \wedge X_{D_{a}}$, where $X_{D_{a}}$ is the linear vector field associated to the linear map $D_{a} \in \operatorname{End}(V)$, and $X_{a}$ is the constant vector corresponding to $i(a)$. Moreover, we have that $\mathcal{L}_{X_{D_{a}}} \Lambda_{V^{*}}=0$.

In the coordinates $\mu_{0}, \mu_{1}, \ldots, \mu_{n}$ on $A^{\dagger}$ associated to the basis $\left\{e^{\alpha}\right\}$, we have

$$
\left\{\mu_{0}, \mu_{0}\right\}=0 \quad\left\{\mu_{0}, \mu_{j}\right\}=\mu_{k} C_{0 j}^{k} \quad\left\{\mu_{i}, \mu_{j}\right\}=\mu_{k} C_{i j}^{k},
$$


where $C_{0 j}^{k}, C_{i j}^{k}$ are the structure constants introduced above. Therefore, the Poisson tensor reads

$$
\Lambda_{A}^{\dagger}=\frac{1}{2} \mu_{k} C_{i j}^{k} \frac{\partial}{\partial \mu_{i}} \wedge \frac{\partial}{\partial \mu_{j}}+\mu_{k} C_{0 j}^{k} \frac{\partial}{\partial \mu_{0}} \wedge \frac{\partial}{\partial \mu_{j}},
$$

where the first term is $\Lambda_{V}$ and the second one is $X_{a} \wedge X_{D_{a}}$ with $a=O$, the origin.

\section{Exterior algebra over an affine space}

In 20] we have defined the concept of differential forms on sections of an affine bundle. We aim in this section to establish at the algebraic level, the relation with ordinary forms on the vector extension. We will re-state in the algebraic setting the definition of a $k$-form, $k \geq 1$.

Definition 2. $A$-form on an affine space $A$ is a map $\omega: A \times \cdots \times A \rightarrow \mathbb{R}$ for which there exists a $k$-form $\boldsymbol{\omega}$ on the associated vector space $V$, and a map $\omega_{0}: A \times V \times \cdots \times V \rightarrow \mathbb{R}$ with the following properties

1. $\omega_{0}$ is skew-symmetric and linear in its $k-1$ vector arguments.

2. For every $a \in A$ and for every $\boldsymbol{v}_{1}, \boldsymbol{v}_{2}, \ldots, \boldsymbol{v}_{k} \in V$, we have

$$
\omega_{0}\left(a+\boldsymbol{v}_{1}, \boldsymbol{v}_{2}, \ldots, \boldsymbol{v}_{k}\right)=\omega_{0}\left(a, \boldsymbol{v}_{2}, \ldots, \boldsymbol{v}_{k}\right)+\boldsymbol{\omega}\left(\boldsymbol{v}_{1}, \boldsymbol{v}_{2}, \ldots, \boldsymbol{v}_{k}\right) .
$$

3. For every $a_{1}, \ldots, a_{k} \in A$, if we choose an arbitrary $a_{0} \in A$ and put $a_{i}=a_{0}+\boldsymbol{v}_{i}$, we have

$$
\omega\left(a_{1}, \ldots, a_{k}\right)=\sum_{j=1}^{k}(-1)^{j+1} \omega_{0}\left(a_{0}, \boldsymbol{v}_{1}, \ldots, \widehat{\boldsymbol{v}}_{j}, \ldots, \boldsymbol{v}_{k}\right)+\boldsymbol{\omega}\left(\boldsymbol{v}_{1}, \boldsymbol{v}_{2}, \ldots, \boldsymbol{v}_{k}\right) .
$$

We next show that a $k$-form on $A$ is just the pull-back by the canonical immersion of a $k$-form on $\tilde{A}$, in other words, $\omega$ is a $k$-form if we have

$$
\omega\left(a_{1}, \ldots, a_{k}\right)=\tilde{\omega}\left(i\left(a_{1}\right), \ldots, i\left(a_{k}\right)\right),
$$

for some ordinary exterior $k$-form $\tilde{\omega}$ on the vector space $\tilde{A}$.

Proposition 3. If $\tilde{\omega}$ is a $k$-form on $\tilde{A}$ then $\omega=i^{*} \tilde{\omega}$ is a $k$-form on the affine space $A$. Conversely, given a $k$-form on the affine space $A$, there exists a unique $k$-form $\tilde{\omega}$ on $\tilde{A}$ such that $\omega=i^{*} \tilde{\omega}$.

Proof. For a given $k$-form $\tilde{\omega}$ on $\tilde{A}$ we define the maps

$$
\begin{aligned}
& \omega\left(a_{1}, \ldots, a_{k}\right)=\tilde{\omega}\left(i\left(a_{1}\right), \ldots, i\left(a_{k}\right)\right), \\
& \omega_{0}\left(a, \boldsymbol{v}_{2}, \ldots, \boldsymbol{v}_{k}\right)=\tilde{\omega}\left(i(a), \boldsymbol{i}\left(\boldsymbol{v}_{2}\right) \ldots, \boldsymbol{i}\left(\boldsymbol{v}_{k}\right)\right), \\
& \boldsymbol{\omega}\left(\boldsymbol{v}_{1} \ldots, \boldsymbol{v}_{k}\right)=\tilde{\omega}\left(\boldsymbol{i}\left(\boldsymbol{v}_{1}\right) \ldots, \boldsymbol{i}\left(\boldsymbol{v}_{k}\right)\right) .
\end{aligned}
$$


Then, conditions 1 and 2 in the definition above are trivially satisfied. Moreover, if we fix $a_{0} \in A$ and write $a_{i}=a_{0}+\boldsymbol{v}_{i}$, then by skew-symmetry of $\tilde{\omega}$ we have

$$
\begin{aligned}
\omega\left(a_{1}, \ldots, a_{k}\right) & =\tilde{\omega}\left(i\left(a_{1}\right), \ldots, i\left(a_{k}\right)\right) \\
& =\tilde{\omega}\left(i\left(a_{0}\right)+\boldsymbol{i}\left(\boldsymbol{v}_{1}\right), \ldots, i\left(a_{0}\right)+\boldsymbol{i}\left(\boldsymbol{v}_{k}\right)\right) \\
& =\sum_{j=1}^{k}(-1)^{j+1} \tilde{\omega}\left(i\left(a_{0}\right), \boldsymbol{i}\left(\boldsymbol{v}_{1}\right), \ldots, \widehat{\boldsymbol{i}\left(\boldsymbol{v}_{j}\right)}, \ldots, \boldsymbol{i}\left(\boldsymbol{v}_{k}\right)\right)+\tilde{\omega}\left(\boldsymbol{i}\left(\boldsymbol{v}_{1}\right), \ldots, \boldsymbol{i}\left(\boldsymbol{v}_{k}\right)\right) \\
& =\sum_{j=1}^{k}(-1)^{j+1} \omega_{0}\left(a_{0}, \boldsymbol{v}_{1}, \ldots, \widehat{\boldsymbol{v}_{j}}, \ldots, \boldsymbol{v}_{k}\right)+\boldsymbol{\omega}\left(\boldsymbol{v}_{1}, \ldots, \boldsymbol{v}_{k}\right),
\end{aligned}
$$

which proves condition 3 .

Conversely, assume we are given a $k$-form $\omega$ on the affine space $A$ with its associated $\omega_{0}$ and $\boldsymbol{\omega}$. Fixing $a_{0} \in A$, we know that every point $z \in \tilde{A}$ can be written in the form $z=\lambda i\left(a_{0}\right)+\boldsymbol{i}(\boldsymbol{v})$ for $\lambda \in \mathbb{R}$ and $\boldsymbol{v} \in V$. We define the map $\tilde{\omega}$ by

$$
\begin{aligned}
\tilde{\omega}\left(z_{1}, \ldots, z_{k}\right) & =\tilde{\omega}\left(\lambda_{1} i\left(a_{0}\right)+\boldsymbol{i}\left(\boldsymbol{v}_{1}\right), \ldots, \lambda_{k} i\left(a_{0}\right)+\boldsymbol{i}\left(\boldsymbol{v}_{k}\right)\right) \\
& =\sum_{j=1}^{k}(-1)^{j+1} \lambda_{j} \omega_{0}\left(a_{0}, \boldsymbol{v}_{1}, \ldots, \widehat{\boldsymbol{v}_{j}}, \ldots, \boldsymbol{v}_{k}\right)+\boldsymbol{\omega}\left(\boldsymbol{v}_{1}, \ldots, \boldsymbol{v}_{k}\right) .
\end{aligned}
$$

By virtue of 1 and 3 , it follows that $\tilde{\omega}$ is multi-linear and skew-symmetric, i.e. it is a $k$-form on $\tilde{A}$. Moreover, $\tilde{\omega}\left(z_{1}, \ldots, z_{k}\right)$ is independent of the choice of the point $a_{0}$. Indeed, if we choose a different point $a_{0}^{\prime}=a_{0}+\boldsymbol{w}$, then $z_{j}=\lambda_{j} i\left(a_{0}^{\prime}\right)+\boldsymbol{v}_{j}^{\prime}$ with $\boldsymbol{v}_{j}^{\prime}=\boldsymbol{v}_{j}-\lambda_{j} \boldsymbol{w}$, and applying the definition above we get

$$
\begin{aligned}
\tilde{\omega}\left(z_{1}, \ldots, z_{k}\right)= & \tilde{\omega}\left(\lambda_{1} i\left(a_{0}^{\prime}\right)+\boldsymbol{i}\left(\boldsymbol{v}_{1}^{\prime}\right), \ldots, \lambda_{k} i\left(a_{0}^{\prime}\right)+\boldsymbol{i}\left(\boldsymbol{v}_{k}^{\prime}\right)\right) \\
= & \sum_{j=1}^{k}(-1)^{j+1} \lambda_{j} \omega_{0}\left(a_{0}^{\prime}, \boldsymbol{v}_{1}^{\prime}, \ldots, \widehat{\boldsymbol{v}_{j}^{\prime}}, \ldots, \boldsymbol{v}_{k}^{\prime}\right)+\boldsymbol{\omega}\left(\boldsymbol{v}_{1}^{\prime}, \ldots, \boldsymbol{v}_{k}^{\prime}\right) \\
= & \sum_{j=1}^{k}(-1)^{j+1} \lambda_{j} \omega_{0}\left(a_{0}+\boldsymbol{w}, \boldsymbol{v}_{1}-\lambda_{1} \boldsymbol{w}, \ldots, \widehat{\boldsymbol{v}_{j}}, \ldots, \boldsymbol{v}_{k}-\lambda_{k} \boldsymbol{w}\right) \\
& \quad+\boldsymbol{\omega}\left(\boldsymbol{v}_{1}-\lambda_{1} \boldsymbol{w}, \ldots, \boldsymbol{v}_{k}-\lambda_{k} \boldsymbol{w}\right) \\
= & \sum_{j=1}^{k}(-1)^{j+1} \lambda_{j} \omega_{0}\left(a_{0}, \boldsymbol{v}_{1}, \ldots, \widehat{\boldsymbol{v}_{j}}, \ldots, \boldsymbol{v}_{k}\right)+\boldsymbol{\omega}\left(\boldsymbol{v}_{1}, \ldots, \boldsymbol{v}_{k}\right)
\end{aligned}
$$

where we have used the properties of $\omega_{0}$ and $\boldsymbol{\omega}$.

The form $\tilde{\omega}$ is unique, since, if $\tilde{\theta}$ is a $k$-form on $\tilde{A}$ such that $i^{*} \tilde{\theta}=0$, then it follows that the associated $\theta_{0}$ and $\boldsymbol{\theta}$ vanish from where we deduce that $\tilde{\theta}=0$.

Once a reference frame has been fixed on $A$, a 1 -form $\tilde{\omega}$ on $\tilde{A}$ is of the form $\tilde{\omega}=\omega_{0} e^{0}+\omega_{i} e^{i}$, and then the local representation of $\omega=i^{*} \tilde{\omega}$ looks exactly the same. 
More generally, a p-form $\tilde{\omega}$ on $\tilde{A}$ is of the form

$$
\tilde{\omega}=\frac{1}{p !} \sum_{\mu_{1}, \ldots, \mu_{p}=0}^{n} \omega_{\mu_{1} \cdots \mu_{p}} e^{\mu_{1}} \wedge \cdots \wedge e^{\mu_{p}}
$$

and thus

$$
\omega=\frac{1}{(p-1) !} \omega_{0 i_{1} \cdots i_{p-1}} e^{0} \wedge e^{i_{1}} \wedge \cdots \wedge e^{i_{p-1}}+\frac{1}{p !} \omega_{i_{1} \cdots i_{p}} e^{i_{1}} \wedge \cdots \wedge e^{i_{p}},
$$

where the first term corresponds to the sum of terms involving $\omega_{0}$ and the second to $\boldsymbol{\omega}$.

\section{$5 \quad$ Lie algebroid structures over affine bundles}

Let $\tau: E \rightarrow M$ be an affine bundle with associated vector bundle $\tau: V \rightarrow M$. We consider the bundle $\tau^{\dagger}: E^{\dagger} \rightarrow M$ whose fibre over $m \in M$ is the extended dual $E_{m}^{\dagger}$ of the fibre $E_{m}$. We also consider the dual bundle $\tilde{\tau}: \tilde{E} \rightarrow M$, whose fibre at $m$ is $\tilde{E}_{m}=\left(E_{m}^{\dagger}\right)^{*}$. At every point $m$ we have the exact sequence of vector spaces

$$
0 \longrightarrow V_{m} \stackrel{\boldsymbol{i}_{m}}{\longrightarrow} \tilde{E}_{m} \stackrel{j_{m}}{\longrightarrow} \mathbb{R} \longrightarrow 0
$$

and therefore an exact sequence of vector bundles over $M$

$$
0 \longrightarrow V \stackrel{i}{\longrightarrow} \tilde{E} \stackrel{j}{\longrightarrow} M \times \mathbb{R} \longrightarrow 0 .
$$

On the other hand there is the canonical immersion $i: E \rightarrow \tilde{E}$, so that $i\left(E_{m}\right)=$ $j^{-1}((m, 1))$.

By taking sections, we have the exact sequence of real vector spaces (and $C^{\infty}(M)$-modules)

$$
0 \longrightarrow \operatorname{Sec}(V) \stackrel{i}{\longrightarrow} \operatorname{Sec}(\tilde{E}) \stackrel{j}{\longrightarrow} C^{\infty}(M) \longrightarrow 0 .
$$

and an inclusion $i: \operatorname{Sec}(E) \rightarrow \operatorname{Sec}(\tilde{E})$, whereby we make no notational distinction between the bundle maps and the induced maps of sections (i.e. if $\sigma$ is a section and $r$ is a bundle map over the identity, we write $r(\sigma)$ instead of $r \circ \sigma$.). It follows that if we fix a section $\sigma$ of $E$ then we have a splitting of the above sequence and any section $\zeta$ of $\tilde{E}$ can be written as $\zeta=f i(\sigma)+\boldsymbol{i}(\boldsymbol{\eta})$, for some section $\boldsymbol{\eta}$ of $V$ and where $f=j(\zeta)$.

Also, since $V$ is the vector bundle associated to the affine bundle $E$, we have that $\operatorname{Sec}(V)$ is the (real) vector space associated to the affine space $\operatorname{Sec}(E)$.

In 20] we defined the concept of Lie algebroid structure on the class of affine bundles whose base manifold is further fibred over $\mathbb{R}$. Here we start with a more general definition, which in the light of the previous section can be expressed as follows. 
Definition 3. A Lie algebroid structure on $E$ consists of a Lie algebra structure on the (real) affine space of sections of $E$ together with an affine map $\rho: E \rightarrow$ $T M$ (the anchor), satisfying the following compatibility condition

$$
D_{\sigma}(f \boldsymbol{\zeta})=\rho(\sigma)(f) \boldsymbol{\zeta}+f D_{\sigma} \boldsymbol{\zeta},
$$

for every $\sigma \in \operatorname{Sec}(E), \zeta \in \operatorname{Sec}(V)$ and $f \in C^{\infty}(M)$, and where $D_{\sigma}$ is the action $\sigma \mapsto D_{\sigma}$ of $\operatorname{Sec}(E)$ on $\operatorname{Sec}(V)$.

The compatibility condition ensures that the association $\sigma \mapsto D_{\sigma}$, which acts by derivations on the real Lie algebra $\operatorname{Sec}(V)$, also acts by derivations on the $C^{\infty}(M)$-module $\operatorname{Sec}(V)$.

The anchor map $\rho$ extends to a linear map $\tilde{\rho}: \tilde{E} \rightarrow T M$, which we will describe in more detail below. It is of interest, however, to observe now already that the map $i: V \rightarrow \tilde{E}$ is a morphism of Lie algebroids, since we have

$$
\left[\boldsymbol{i}\left(\boldsymbol{\eta}_{1}\right), \boldsymbol{i}\left(\boldsymbol{\eta}_{2}\right)\right]=\boldsymbol{i}\left(\left[\boldsymbol{\eta}_{1}, \boldsymbol{\eta}_{2}\right]\right) \text { and } \tilde{\rho} \circ \boldsymbol{i}=\boldsymbol{\rho},
$$

where $\boldsymbol{\rho}$ is the linear part of $\rho$. On the contrary, the map $j: \tilde{E} \rightarrow M \times \mathbb{R}$ is NOT a morphism of Lie algebroids, since we have that $j\left(\left[\zeta_{1}, \zeta_{2}\right]\right)=\tilde{\rho}\left(\zeta_{1}\right) f_{2}-\tilde{\rho}\left(\zeta_{2}\right) f_{1}$, while $\left[j\left(\zeta_{1}\right), j\left(\zeta_{2}\right)\right]=0$ since the fibres of $M \times \mathbb{R}$ are 1-dimensional.

The affine Lie algebroid structure we studied in $[20$ is the case that $M$ is further fibred over the real line $\pi: M \rightarrow \mathbb{R}$ and the anchor map $\rho$ takes values in $J^{1} M$. Notice that such an extra fibration is not generally available, not even locally. For instance, if we take any affine bundle $\tau: E \rightarrow M$ with the trivial Lie algebroid structure (null bracket and anchor) then there is no fibration over $\mathbb{R}$ such that the image of $\rho$ is in $J^{1} M$, since the vectors in $i\left(J^{1} M\right)$ are non-zero.

The following result shows that one can alternatively define an affine Lie algebroid structure on $E$ as a vector Lie algebroid structure ([, ], $\tilde{\rho})$ on $\tilde{E}$ such that the bracket of two sections in the image of $i$ belongs to the image of $\boldsymbol{i}$.

Theorem 2. A Lie algebroid structure on the vector bundle $\tilde{\tau}: \tilde{E} \rightarrow M$ which is such that the bracket of sections in the image of $i$ lies in the image of $\boldsymbol{i}$ defines a Lie algebroid structure on the affine bundle $\tau: E \rightarrow M$, whereby the brackets and maps are determined by the following relations:

$$
\begin{aligned}
\boldsymbol{i}\left(\left[\boldsymbol{\eta}_{1}, \boldsymbol{\eta}_{2}\right]\right) & =\left[\boldsymbol{i}\left(\boldsymbol{\eta}_{1}\right), \boldsymbol{i}\left(\boldsymbol{\eta}_{2}\right)\right] \\
\boldsymbol{i}\left(D_{\sigma} \boldsymbol{\eta}\right) & =[i(\sigma), \boldsymbol{i}(\boldsymbol{\eta})] \\
\rho(\sigma) & =\tilde{\rho}(i(\sigma)) .
\end{aligned}
$$

Conversely, a Lie algebroid structure on the affine bundle $\tau: E \rightarrow M$ extends to a Lie algebroid structure on the vector bundle $\tilde{\tau}: \tilde{E} \rightarrow M$ such that the bracket of sections in the image of $i$ is in the image of $\boldsymbol{i}$. If we fix a section $\sigma$ of $E$ and write sections $\zeta$ of $\tilde{E}$ (locally) in the form $\zeta=f i(\sigma)+\boldsymbol{i}(\boldsymbol{\eta})$ then the anchor and the bracket are given by

$$
\begin{gathered}
\tilde{\rho}(\zeta)=f \rho(\sigma)+\boldsymbol{\rho}(\boldsymbol{\eta}) \\
{\left[\zeta_{1}, \zeta_{2}\right]=\left(\tilde{\rho}\left(\zeta_{1}\right)\left(f_{2}\right)-\tilde{\rho}\left(\zeta_{2}\right)\left(f_{1}\right)\right) i(\sigma)+\boldsymbol{i}\left(\left[\boldsymbol{\eta}_{1}, \boldsymbol{\eta}_{\mathbf{2}}\right]+f_{1} D_{\sigma} \boldsymbol{\eta}_{2}-f_{2} D_{\sigma} \boldsymbol{\eta}_{1}\right) .}
\end{gathered}
$$


Proof. The verification of the above statements is straightforward but rather lengthy. We limit ourselves to checking that the compatibility conditions between brackets and anchors are satisfied. For the first part, we find

$$
\begin{aligned}
i\left(D_{\sigma}(f \boldsymbol{\eta})\right) & =[i(\sigma), \boldsymbol{i}(f \boldsymbol{\eta})]=[i(\sigma), f \boldsymbol{i}(\boldsymbol{\eta})] \\
& =\tilde{\rho}(i(\sigma))(f) \boldsymbol{i}(\boldsymbol{\eta})+f[i(\sigma), \boldsymbol{i}(\boldsymbol{\eta})]=\boldsymbol{i}\left(\rho(\sigma)(f) \boldsymbol{\eta}+f D_{\sigma}(\boldsymbol{\eta})\right),
\end{aligned}
$$

form which it follows that $D_{\sigma}(f \boldsymbol{\eta})=\rho(\sigma)(f) \boldsymbol{\eta}+f D_{\sigma}(\boldsymbol{\eta})$.

For the converse, observe that

$$
\begin{aligned}
{\left[\zeta_{1}, f \zeta_{2}\right]-f\left[\zeta_{1}, \zeta_{2}\right] } & =f_{2} \tilde{\rho}\left(\zeta_{1}\right)(f) i(\sigma)+\boldsymbol{i}\left(f_{1} \rho(\sigma)(f) \boldsymbol{\eta}_{\mathbf{2}}+\boldsymbol{\rho}\left(\boldsymbol{\eta}_{\mathbf{1}}\right)(f) \boldsymbol{\eta}_{2}\right) \\
& =\tilde{\rho}\left(\zeta_{1}\right)(f) f_{2} i(\sigma)+\left(f_{1} \rho(\sigma)+\boldsymbol{\rho}\left(\boldsymbol{\eta}_{\mathbf{1}}\right)\right)(f) \boldsymbol{i}\left(\boldsymbol{\eta}_{2}\right) \\
& =\tilde{\rho}\left(\zeta_{1}\right)(f) \zeta_{2},
\end{aligned}
$$

which is the required compatibility condition.

In coordinates, if $x^{i}$ denote coordinates on $M$ and $y^{\alpha}$ fibre coordinates on $E$ with respect to some local frame $\left(e_{0},\left\{\boldsymbol{e}_{\alpha}\right\}\right)$ of sections of $E$, then we have

$$
\begin{aligned}
\rho\left(e_{0}+y^{\alpha} \boldsymbol{e}_{\alpha}\right) & =\left(\rho_{0}^{i}+\rho_{\alpha}^{i} y^{\alpha}\right) \frac{\partial}{\partial x^{i}}, \\
{\left[\boldsymbol{e}_{\alpha}, \boldsymbol{e}_{\beta}\right] } & =C_{\alpha \beta}^{\gamma} \boldsymbol{e}_{\gamma}, \\
D_{e_{0}} \boldsymbol{e}_{\beta} & =C_{0 \beta}^{\gamma} \boldsymbol{e}_{\gamma},
\end{aligned}
$$

for some functions $\rho_{0}^{i}, \rho_{\alpha}^{i}, C_{0 \beta}^{\gamma}$ and $C_{\alpha \beta}^{\gamma}$ on $M$.

Taking the local basis of sections of $\tilde{E}$, associated to the above frame, it follows that

$$
\tilde{\rho}\left(y^{0} e_{0}+y^{\alpha} e_{\alpha}\right)=\left(\rho_{0}^{i} y^{0}+\rho_{\alpha}^{i} y^{\alpha}\right) \frac{\partial}{\partial x^{i}},
$$

and the bracket is determined by

$$
\left[e_{0}, e_{0}\right]=0 \quad\left[e_{0}, e_{\beta}\right]=C_{0 \beta}^{\gamma} e_{\gamma} \quad\left[e_{\alpha}, e_{\beta}\right]=C_{\alpha \beta}^{\gamma} e_{\gamma} .
$$

As a final remark we mention that the orbits of the Lie algebroid $\tau: V \rightarrow M$ are subsets of the orbits of the algebroid $\tilde{\tau}: \tilde{E} \rightarrow M$ and they are equal if and only if there exists a section $\sigma$ of $E$ such that $\rho(\sigma)$ is in the image of $\boldsymbol{\rho}$.

\section{Exterior differential and Poisson structure}

Now that we have proved that a Lie algebroid structure on an affine bundle is equivalent to a Lie algebroid structure on $\tilde{E}$, we can define the exterior differential operator on $E$ by pulling back the exterior differential on $\tilde{E}$. More precisely, given a $k$-form $\omega$ on the affine bundle $E$ we know that there exists a unique $\tilde{\omega}$ on $\tilde{E}$ such that $\omega=i^{*}(\tilde{\omega})$. Then we define $d \omega$ as the $(k+1)$-form given by

$$
d \omega=i^{*}(d \tilde{\omega}) .
$$


It is easy to see that this definition is equivalent to the one given in [20] (at least when $M$ is fibred over $\mathbb{R}$ ).

The definition given here has some clear advantages. For instance, the property $d^{2}=0$ which was rather difficult to prove in [20], becomes evident now:

$$
d^{2} \omega=d\left(d\left(i^{*} \tilde{\omega}\right)\right)=d\left(i^{*} d \tilde{\omega}\right)=i^{*}\left(d^{2} \tilde{\omega}\right)=0 .
$$

The differential $\boldsymbol{d}$ on the Lie algebroid $V$ is also related to the differential on $\tilde{E}$; for every $k$-form $\tilde{\omega}$ on $E$ we have that

$$
\boldsymbol{d i}^{*} \tilde{\omega}=\boldsymbol{i}^{*}(d \tilde{\omega})
$$

which in fact simply expresses that $\boldsymbol{i}$ is a morphism of Lie algebroids. Hence, one can find the differential of a form $\boldsymbol{\omega}$ on $V$ by choosing a $k$-form $\tilde{\omega}$ on $\tilde{E}$ such that $\boldsymbol{i}^{*} \tilde{\boldsymbol{\omega}}=\boldsymbol{\omega}$ and then obtain $\boldsymbol{d} \boldsymbol{\omega}$ as $\boldsymbol{i}^{*}(d \tilde{\omega})$. That this does not depend on the choice of $\tilde{\omega}$ is expressed in equivalent terms by the following result.

Proposition 4. The ideal $\mathcal{I}=\left\{\tilde{\omega} \mid \boldsymbol{i}^{*} \tilde{\omega}=0\right\}$ is a differential ideal, i.e. $d \mathcal{I} \subset \mathcal{I}$.

Proof. If $\boldsymbol{i}^{*} \tilde{\omega}=0$, then the same is true for $d \tilde{\omega}$ since $\boldsymbol{i}^{*}(d \tilde{\omega})=\boldsymbol{d} \boldsymbol{i}^{*}(\tilde{\omega})=0$.

The ideal $\mathcal{I}$ is generated by the 1 -form $e^{0}, \mathcal{I}=\left\{e^{0} \wedge \theta \mid \theta\right.$ is a form on $\left.\tilde{E}\right\}$ so that $d e^{0}$ belongs to $\mathcal{I}$. The following result shows that $e^{0}$ in fact is $d$-closed and that this property characterises affine structures.

Theorem 3. A Lie algebroid structure on $\tilde{E}$ restricts to a Lie algebroid structure on the affine bundle $E$ if and only if the exterior differential satisfies $d e^{0}=0$.

Proof. Indeed, taking two sections $\sigma_{1}$ and $\sigma_{2}$ of $E$ we have

$$
\begin{aligned}
d e^{0}\left(i\left(\sigma_{1}\right), i\left(\sigma_{2}\right)\right) & =\tilde{\rho}\left(\sigma_{1}\right)\left\langle e^{0}, i\left(\sigma_{2}\right)\right\rangle-\tilde{\rho}\left(\sigma_{2}\right)\left\langle e^{0}, i\left(\sigma_{1}\right)\right\rangle-\left\langle e^{0},\left[i\left(\sigma_{1}\right), i\left(\sigma_{2}\right)\right]\right\rangle \\
& =-\left\langle e^{0},\left[i\left(\sigma_{1}\right), i\left(\sigma_{2}\right)\right]\right\rangle
\end{aligned}
$$

It follows that $\left[i\left(\sigma_{1}\right), i\left(\sigma_{2}\right)\right]$ is in $\operatorname{Im}(\boldsymbol{i})=\operatorname{Ker}\left(e^{0}\right)$ if and only if $d e^{0}$ vanishes on the image of $i$, which spans $\tilde{E}$.

In coordinates, the exterior differential operator is determined by

$$
d f=\rho_{0}^{i} \frac{\partial f}{\partial x^{i}} e^{0}+\rho_{\alpha}^{i} \frac{\partial f}{\partial x^{i}} e^{\alpha}, \quad \text { for } f \in C^{\infty}(M)
$$

and

$$
d e^{0}=0, \quad d e^{\gamma}=-C_{0 \beta}^{\gamma} e^{0} \wedge e^{\beta}-\frac{1}{2} C_{\alpha \beta}^{\gamma} e^{\alpha} \wedge e^{\beta} .
$$

In the special case that $\rho(E) \subset J^{1} M$ ( $M$ fibred over $\mathbb{R}$ ), we have that $d t=e^{0}$ so that $e^{0}$ is not only closed but also exact. In fact, this is the condition for a Lie algebroid structure on an affine bundle to have a 1-jet-valued anchor. Indeed, if there exists an $f \in C^{\infty}(M)$ such that $d f=e^{0}$, then the partial derivatives of 
$f$ cannot simultaneously vanish, hence $f$ defines a local fibration and then for any section $\sigma$ of $E$ we have that $\rho(\sigma) f=\langle d f, \sigma\rangle=\left\langle e^{0}, \sigma\right\rangle=1$, which is the condition for the anchor being 1-jet-valued.

When we have a Lie algebroid structure on $\tilde{E}$, there is a Poisson bracket on the dual bundle $\tilde{E}^{*}=E^{\dagger}$. Any section $\zeta$ of $\tilde{E}$, and in particular any section of $E$, determines a linear function $\widehat{\zeta}$ on $E^{\dagger}$ by

$$
\widehat{\zeta}(\varphi)=\left\langle\zeta_{m}, \varphi\right\rangle \quad \text { for every } \varphi \in E_{m}^{\dagger} .
$$

Then the Poisson bracket is determined by the condition

$$
\left\{\widehat{\zeta}_{1}, \widehat{\zeta}_{2}\right\}=\widehat{\left[\zeta_{1}, \zeta_{2}\right]}
$$

which for consistency (using linearity and the Leibnitz rule) requires that we put

$$
\{\widehat{\zeta}, g\}=\tilde{\rho}(\zeta)(g), \quad \text { and } \quad\{f, g\}=0,
$$

for $f$ and $g$ functions on $M$.

It is of some interest to mention yet another characterisation of the result described in Theorem 2. The above Poisson bracket in fact is determined by the bracket of linear functions coming from sections of $E$, since these span the set of all linear functions on $E^{\dagger}$. But the bracket of sections of $E$ is a section of $V$; it follows that the corresponding Poisson brackets are independent of the coordinate $\mu_{0}$, and therefore, $\frac{\partial}{\partial \mu_{0}}$ is a symmetry of the Poisson tensor. Conversely, it is obvious that the latter symmetry property will imply that the bracket of sections in the image of $i$ belongs to the image of $i$.

It should be noticed that, in general, the Poisson structure we have defined is not an extension of a Poisson structure on $M \times \mathbb{R}$ by the one on $V^{*}$. Indeed, the map $k: E^{\dagger} \rightarrow V^{*}$ is a Poisson map (since it is the dual of $\boldsymbol{i}$ and this is a Lie algebroid morphism), but the map $l: M \times \mathbb{R} \rightarrow E^{\dagger}$ is not a Poisson map, except if $\boldsymbol{\rho}=0$. This is equivalent to the dual mapping $l^{*}=j$ not being a Lie algebroid morphism. To prove this, we first show that the brackets $\{\widehat{\boldsymbol{\eta}}, f\} \circ l$ and $\{\widehat{\boldsymbol{\eta}} \circ l, f \circ l\}$ are different, except when $\boldsymbol{\rho}=0$. Indeed, we have that $l(m, p)=p e^{0}$, so that $\widehat{\boldsymbol{\eta}} \circ l=0$ and hence $\{\widehat{\boldsymbol{\eta}} \circ l, f \circ l\}=0$. On the other hand, $\{\widehat{\boldsymbol{\eta}}, f\} \circ l=\boldsymbol{\rho}(\boldsymbol{\eta})(f) \circ l=\boldsymbol{\rho}(\boldsymbol{\eta})(f)$, since $\boldsymbol{\rho}(\boldsymbol{\eta})(f)$ is a function on $M$. Therefore, the two brackets are equal if and only if $\boldsymbol{\rho}=0$. Similarly, one can calculate the brackets $\left\{\widehat{e_{0}}, f\right\} \circ l=\left\{\mu_{0}, f\right\}$ and $\left\{\widehat{e_{0}} \circ l, f \circ l\right\}=\rho\left(e_{0}\right)(f)$. This imposes that the Poisson tensor on $M \times \mathbb{R}$ has to be $\Lambda=\frac{\partial}{\partial \mu_{0}} \wedge X_{0}$, with $X_{0}=\rho\left(e_{0}\right)$. (Notice that $\rho\left(e_{0}\right)=\rho(\sigma)$ for any section $\sigma$ of $E$.) The other brackets vanish, so there are no further conditions.

In coordinates, we have

$$
\begin{array}{lll}
\left\{x^{i}, x^{j}\right\}=0 & \left\{\mu_{0}, x^{i}\right\}=\rho_{0}^{i} & \left\{\mu_{\alpha}, x^{i}\right\}=\rho_{\alpha}^{i} \\
\left\{\mu_{0}, \mu_{\beta}\right\}=C_{0 \beta}^{\gamma} \mu_{\gamma} & \left\{\mu_{\alpha}, \mu_{\beta}\right\}=C_{\alpha \beta}^{\gamma} \mu_{\gamma} &
\end{array}
$$


and therefore the Poisson tensor is

$$
\begin{aligned}
\Lambda_{E^{\dagger}}=\rho_{\alpha}^{i} \frac{\partial}{\partial \mu_{\alpha}} \wedge \frac{\partial}{\partial x^{i}} & +\frac{1}{2} \mu_{\gamma} C_{\alpha \beta}^{\gamma} \frac{\partial}{\partial \mu_{\alpha}} \wedge \frac{\partial}{\partial \mu_{\beta}}+ \\
+ & \frac{\partial}{\partial \mu_{0}} \wedge\left(\rho_{0}^{i} \frac{\partial}{\partial x^{i}}+\mu_{\gamma} C_{0 \beta}^{\gamma} \frac{\partial}{\partial \mu_{\beta}}\right) .
\end{aligned}
$$

\section{$7 \quad$ Examples}

The canonical affine Lie algebroid The canonical example of a Lie algebroid over an affine bundle is the first jet bundle $J^{1} M \rightarrow M$ to a manifold $M$ fibered over the real line $\pi: M \rightarrow \mathbb{R}$. The elements of the manifold $J^{1} M$ are equivalence classes $j_{t}^{1} \gamma$ of sections $\gamma$ of the bundle $\pi: M \rightarrow \mathbb{R}$, where two sections are equivalent if they have first order contact at the point $t$. It is an affine bundle whose associated vector bundle is $\operatorname{Ver}(\pi)$ the set of vectors tangent to $M$ which are vertical over $\mathbb{R}$. In this case it is well-known that $J^{1} M^{\dagger}=T^{*} M$, and therefore $\widetilde{J^{1} M}=T M$. The canonical inmersion is given by

$$
i\left(j_{t}^{1} \gamma\right)=\dot{\gamma}(t)
$$

i.e. it maps the 1-jet of the section $\gamma$ at the point $t$ to the vector tangent to $\gamma$ at the point $t$. In coordinates, if $j_{t}^{1} \gamma$ has coordinates $(t, x, v)$ then

$$
i(t, x, v)=\left.\frac{\partial}{\partial t}\right|_{(t, x)}+\left.v^{i} \frac{\partial}{\partial x^{i}}\right|_{(t, x)}
$$

An element $w$ of the associated vector bundle $\operatorname{Ver}(\pi)$ is of the form

$$
w=\left.w^{i} \frac{\partial}{\partial x^{i}}\right|_{(t, x)}
$$

The bracket of sections of $J^{1} M$ is defined precisely by means of the above identification of 1 -jets with vectors $v \in T M$ which projects to the vector $\partial / \partial t$. In coordinates a section $X$ of $J^{1} M$ is identified with the vector field

$$
X=\frac{\partial}{\partial t}+X^{i}(t, x) \frac{\partial}{\partial x^{i}}
$$

and the bracket is

$$
[X, Y]=\left\{X\left(Y^{i}\right)-Y\left(X^{i}\right)\right\} \frac{\partial}{\partial x^{i}}
$$

which is obviously a section of the vector bundle.

Affine distributions An affine $E$ sub-bundle of $J^{1} M$ is involutive if the bracket of sections of the sub-bundle is a section of the associated vector bundle. Therefore, taking as anchor the natural inclusion into $T M$ and as bracket the restriction of the bracket in $J^{1} M$ to $E$ we have an affine Lie algebroid structure on $E$. 
Lie algebra structures on affine spaces We consider the case in which the manifold $M$ reduces to one point $M=\{m\}$. Thus our affine bundle is $E=\{m\} \times A$ and the associated vector bundle is $W \equiv\{m\} \times V$ for some affine space $A$ over the vector space $V$. Then, a Lie algebroid structure over the affine bundle $E$ is just an affine Lie algebra structure over $A$. Indeed, every section of $E$ and of $W$ is determined by a point in $A$ and $V$, respectively. The anchor must vanishes since $T M=\left\{0_{m}\right\}$, so it does not carry any additional information.

Trivial affine algebroids By a trivial affine space we mean just a point $A=\{O\}$, and the associated vector space is the trivial one $V=\{0\}$. The extended affine dual of $A$ is $A^{\dagger}=\mathbb{R}$ since the only affine maps defined on a space of just a point are the constant maps. It follows that the extended bi-dual is $\tilde{A}=\mathbb{R}$

Given a manifold $M$, we consider the affine bundle $E=M \times\{O\}$ with associated vector bundle $V=M \times\{0\}$. On $V$ we consider the trivial bracket $[]=$,0 and the anchor $\boldsymbol{\rho}=0$, and as derivation $D_{O}$ we also take $D_{O}=0$. Now, to construct a Lie algebroid structure on $E$, we take an arbitrary vector field $X_{0}$ on $M$ as given and define the map $\rho: E \rightarrow T M$ by $\rho(m, O)=X_{0}(m)$. Then it follows that $\rho$ is compatible with $D_{O}$.

The extended dual of $E$ is $E^{\dagger}=M \times \mathbb{R}$ and the extended bi-dual is $\tilde{E}=$ $M \times \mathbb{R}$. We therefore have one section $e^{0}$ spanning the set of sections of $E^{\dagger}$, and the dual element $e_{0}$ (which is just the image under the canonical immersion of the constant section of value 0 .)

We want to study the associated exterior differential operator and Poisson bracket.

For the exterior differential operator, since we have 1-dimensional fibre on $E^{\dagger}$ it follows that $d e^{0}=0$. On functions $f \in C^{\infty}(M)$ we have $d f=\rho\left(e_{0}\right)(f) e^{0}=$ $X_{0}(f) e^{0}$.

For the Poisson structure, since the fibre of $E^{\dagger}$ is 1-dimensional, it is determined by the equation $\left\{\hat{e_{0}}, f\right\}=\rho\left(e_{0}\right)(f)$ and $\hat{e_{0}}=\mu_{0}$. We have that the only non-trivial brackets are $\left\{\mu_{0}, f\right\}=X_{0}(f)$. Therefore, the Poisson tensor is

$$
\Lambda=\frac{\partial}{\partial \mu_{0}} \wedge X_{0}
$$

Quotient by a group If $p: Q \rightarrow M$ is a principal $G$-bundle and $M$ is fibred over $\mathbb{R}$, then $E=J^{1} Q / G \rightarrow M$ is an affine Lie algebroid. The anchor is $\rho\left(\left[j_{t}^{1} \gamma\right]\right)=j_{t}^{1}(p \circ \gamma)$. The bracket is obtained by projecting the bracket on $J^{1} Q$. We have that $\tilde{E}$ is the Atiyah algebroid $=T Q / G$, see for instance [1].

Affine actions of Lie algebras Let $A$ be an affine space endowed with a Lie algebra structure. By an action of $A$ on a manifold $M$ we mean an affine map $\phi: A \rightarrow \mathfrak{X}(M)$, such that $[\phi(a), \phi(b)]=\phi([a, b])$. Then $M \times A \rightarrow M$ has an affine Lie algebroid structure. The anchor is $\rho(m, \xi)=\phi(\xi)(m)$ and the bracket can be defined in terms of constant sections: the bracket of two constant 
sections $\sigma_{i}(m)=\left(m, \xi_{i}\right)$, is the constant section corresponding to the bracket of the values

$$
\left[\sigma_{1}, \sigma_{2}\right](m)=\left(m,\left[\xi_{1}, \xi_{2}\right]_{A}\right) .
$$

If we consider the Lie algebra $\tilde{A}$ then $\tilde{A}$ acts also on the manifold $M$. The extension $\tilde{E}$ is the Lie algebroid associated to the action of $\tilde{A}$.

Poisson manifolds with symmetry Consider a Poisson manifold $(M, \Lambda)$ and an infinitesimal symmetry $Y \in \mathfrak{X}(M)$ of $\Lambda$, that is $\mathcal{L}_{Y} \Lambda=0$. Take $E$ to be $T^{*} M$ with its natural affine structure, where the associated vector bundle is $V=T^{*} M$ itself. On $V$ we consider the Lie algebroid structure defined by the canonical Poisson structure. For a section $\alpha$ of $E$ (i.e. a 1-form on $M$ ) we define the map $D_{\alpha}: \operatorname{Sec}(V) \rightarrow \operatorname{Sec}(V)$ by

$$
D_{\alpha} \beta=\mathcal{L}_{Y} \beta+[\alpha, \beta] .
$$

Since $Y$ is a symmetry of $\Lambda, D_{\alpha}$ is a derivation and clearly satisfies the required compatibility condition. If we further consider the affine anchor $\rho: E \rightarrow T M$, determined by $\rho\left(\alpha_{m}\right)=\Lambda\left(\alpha_{m}\right)+Y_{m}$, then we have a Lie algebroid structure on the affine bundle $E$.

In this case, since there is a distinguished section of $E$ (the zero section), we have that $E^{\dagger}=T M \times \mathbb{R}$ and $\tilde{E}=T^{*} M \times \mathbb{R}$.

Jets of sections in a groupoid Let $G$ be a Lie groupoid over a manifold $M$ with source $\alpha$ and target $\beta$ (the notation is as in $\left[\mathbb{1} \mid\right.$ ). Let $T_{G^{(0)}}^{\alpha} G=\left.\operatorname{ker} T \alpha\right|_{G^{(0)}}$ be the associated Lie algebroid, that is, the set of $\alpha$-vertical vectors at points in $G^{(0)}$ (the set of identities). The anchor is the map $\rho=T \beta$. Assume that $M$ is further fibred over the real line, $\pi: M \rightarrow \mathbb{R}$ and consider the bundle $E=J_{G^{(0)}}^{\alpha} G$ of 1-jets of sections of $\pi \circ \beta$ which are $\alpha$-vertical, at points in $G^{(0)}$. This is an affine bundle whose associated vector bundle is $\left(T_{G^{(0)}}^{\alpha} G\right)^{\text {ver }}$ the set of $(\pi \circ \beta)$-vertical vectors on $T_{G^{(0)}}^{\alpha} G$. If $\boldsymbol{i}$ is the natural inclusion of $\left(T_{G^{(0)}}^{\alpha} G\right)^{\text {ver }}$ into $T_{G^{(0)}}^{\alpha} G$ and we define the map $j: T_{G^{(0)}}^{\alpha} G \rightarrow M \times \mathbb{R}$ by $j(v)=\left(\alpha\left(\tau_{G}(v)\right), t(v)\right)$ (where $t=\pi \circ \beta \circ \tau_{G}$ ), then we have the exact sequence of vector bundles over $M$

$$
0 \longrightarrow\left(T_{G^{(0)}}^{\alpha} G\right)^{\text {ver }} \stackrel{i}{\longrightarrow} T_{G^{(0)}}^{\alpha} G \stackrel{j}{\longrightarrow} M \times \mathbb{R} \longrightarrow 0
$$

and $j^{-1}(M \times\{1\})=J_{G^{(0)}}^{\alpha} G$. Moreover, the bracket of two sections of $J_{G^{(0)}}^{\alpha} G$ is vertical over $\mathbb{R}$ from where it follows that the Lie algebroid structure of $T_{G^{(0)}}^{\alpha} G$ restricts to a Lie algebroid structure on the affine bundle $J_{G^{(0)}}^{\alpha} G$.

\section{Prolongation}

In this section we define the prolongation of a fibre bundle with respect to a (vector) Lie algebroid. We are primarily interested in the prolongation of the bundle $E \rightarrow M$, but we will describe explicitly a more general construction first, since this does not introduce extra complications (see also [8] for generalities). 
Let $\mu: P \rightarrow M$ be a fibre bundle over the manifold $M$ and $F \rightarrow M$ a Lie algebroid with anchor $\rho$. Using notations introduced in [16], we consider the bundle $\mu_{1}: \mathcal{L}^{F} P \rightarrow P$ constructed as follows. The manifold $\mathcal{L}^{F} P$ is the total space of $\rho^{*}(T P)$, the pull-back of $T P$ by $\rho$, i.e.

$$
\mathcal{L}^{F} P=\{(z, V) \in F \times T P \mid \rho(z)=T \mu(V)\} .
$$

The bundle projection we consider, however, is not the usual one

$$
\mu_{2}: \mathcal{L}^{F} P \rightarrow F \quad \mu_{2}(z, V)=z,
$$

but rather $\mu_{1}: \mathcal{L}^{F} P \rightarrow P$, defined by

$$
\mu_{1}(z, V)=\tau_{P}(V)
$$

where $\tau_{P}$ is the tangent bundle projection $T P \rightarrow P$.

A section $X$ of $\mathcal{L}^{F} P$ is of the form $X(p)=(\theta(p), V(p))$, where $\theta$ is a section of $F$ along $\mu$ and $V$ is a vector field on $P$. A section $X$ is projectable if there exists a section $\sigma$ of $F$ such that $\mu_{2} \circ X=\sigma \circ \mu$. It follows that $X$ is projectable if and only if $\theta=\sigma \circ \mu$, and therefore $X$ is of the form $X(p)=(\sigma(m), A(p))$, with $m=\mu(p)$.

The prolongation of $P$ inherits a Lie algebroid structure from the one on $F$ and the one on $T P$. The anchor is the map

$$
\rho^{1}: \mathcal{L}^{F} P \rightarrow T P \quad \rho^{1}(z, V)=V,
$$

and the bracket can be defined in terms of projectable sections as follows. If $X_{1}, X_{2}$ are two projectable sections given by $X_{k}(p)=\left(\sigma_{k}(m), V_{k}(p)\right), k=1,2$, then the bracket $\left[X_{1}, X_{2}\right]$ is the section given by

$$
\left[X_{1}, X_{2}\right](p)=\left(\left[\sigma_{1}, \sigma_{2}\right](m),\left[V_{1}, V_{2}\right](p)\right) .
$$

From this expression it is clear that $\left[X_{1}, X_{2}\right]$ is also a projectable section.

An element $X$ of $\mathcal{L}^{F} P$ is said to be vertical if $\mu_{2}(X)=0$. Therefore it is of the form $(0, V)$, for some vertical vector $V$ on $P$. One should realise, however, that there are sections $X$ of $\mathcal{L}^{F} P$ for which $\rho^{1}(X)$ is a vertical vector on $P$, but $X$ itself is not vertical. Such elements are of the form $(z, V)$, with $z$ in the kernel of $\rho$.

Given a local basis $\left\{e_{a}\right\}$ of sections of $F$ and local coordinates $\left(x^{i}, u^{I}\right)$ on $P$ we can define a basis of sections of $\mathcal{L}^{F} P$, as follows

$$
\begin{aligned}
& \mathcal{X}_{a}(p)=\left(e_{a}(m),\left.\rho_{a}^{i} \frac{\partial}{\partial x^{i}}\right|_{p}\right) \\
& \mathcal{V}_{I}(p)=\left(0_{m}, \frac{\partial}{\partial u^{I}}\right)
\end{aligned}
$$


where $m=\mu(p)$. Thus, any element $Z$ of $\mathcal{L}^{F} P$ at $p$

$$
Z=\left(z^{a} e_{a}(m),\left.\left(\rho_{a}^{i} z^{a}\right) \frac{\partial}{\partial x^{i}}\right|_{p}+\left.v^{I} \frac{\partial}{\partial u^{I}}\right|_{p}\right)
$$

can be represented as

$$
Z=z^{a} \mathcal{X}_{a}+v^{I} \mathcal{V}_{I}
$$

and $\left(x^{i}, u^{I}, z^{a}, v^{I}\right)$ are coordinates on $\mathcal{L}^{F} P$. The brackets of the elements of this basis are

$$
\left[\mathcal{X}_{a}, \mathcal{X}_{b}\right]=C_{a b}^{c} \mathcal{X}_{c} \quad\left[\mathcal{X}_{a}, \mathcal{V}_{J}\right]=0 \quad\left[\mathcal{V}_{I}, \mathcal{V}_{J}\right]=0
$$

and the exterior differential is determined by

$$
d x^{i}=\rho_{a}^{i} \mathcal{X}^{a}, \quad d u^{I}=\mathcal{V}^{I}
$$

and

$$
d \mathcal{X}^{c}=-\frac{1}{2} C_{a b}^{c} \mathcal{X}^{a} \wedge \mathcal{X}^{b} \quad d \mathcal{V}^{I}=0
$$

where $\left\{\mathcal{X}^{c}, \mathcal{V}^{I}\right\}$ denotes a dual basis.

As a first step towards the situation we are most interested in, we consider the case where the Lie algebroid $F$ is either $V$ or $\tilde{E}$. We will show that the Lie algebroid structure on $\mathcal{L}^{\tilde{E}} P$ then is precisely of the kind we are studying in this paper.

Proposition 5. Let I: $\mathcal{L}^{V} P \rightarrow \mathcal{L}^{\tilde{E}} P$ and $J: \mathcal{L}^{\tilde{E}} P \rightarrow P \times \mathbb{R}$ be the maps

$$
\boldsymbol{I}(v, W)=(\boldsymbol{i}(v), W) \quad \text { and } \quad J(z, V)=\left(\mu_{1}(z, V), j_{m}(z)\right) .
$$

Then the following sequence of vector bundles is exact

$$
0 \longrightarrow \mathcal{L}^{V} P \stackrel{\boldsymbol{I}}{\longrightarrow} \mathcal{L}^{\tilde{E}} P \stackrel{J}{\longrightarrow} P \times \mathbb{R} \longrightarrow 0 .
$$

Proof. We clearly have that $J \circ \boldsymbol{I}=0$, so that $\operatorname{Im}(\boldsymbol{I}) \subset \operatorname{Ker}(J)$. Moreover, if $(z, V) \in \operatorname{Ker}(J)$ then $j(z)=0$; hence there exists a $\boldsymbol{v}$ such that $z=\boldsymbol{i}(\boldsymbol{v})$, so that $(z, V)=(\boldsymbol{i}(\boldsymbol{v}), V)=\boldsymbol{I}(\boldsymbol{v}, V)$ is in $\operatorname{Im}(\boldsymbol{I})$.

Therefore, the set of points $\mathcal{J}^{E} P=J^{-1}(P \times\{1\})$ is an affine bundle whose associated vector bundle is $\mathcal{L}^{V} P$. Explicitly, we have

$$
\mathcal{J}^{E} P=\left\{(i(a), V) \in \mathcal{L}^{\tilde{E}} P\right\} \simeq\{(a, V) \in E \times T P \mid \rho(a)=T \mu(V)\} .
$$

Moreover, the Lie algebroid structure of $\mathcal{L}^{\tilde{E}} P$ restricts to $\mathcal{J}^{E} P$, defining therefore a Lie algebroid structure on that affine bundle. To see this, we have to prove that the bracket of sections of $\mathcal{J}^{E} P$ is a section of $\mathcal{L}^{V} P$. For that it is enough to consider projectable sections, since they form a generating set. If $Z_{1}$, $Z_{2}$ are sections of $\mathcal{J}^{E} P$, projecting to sections $\sigma_{1}$ and $\sigma_{2}$ of $E$, then for every $p \in P$ we have

$$
\left[Z_{1}, Z_{2}\right](p)=\left(\left[\sigma_{1}, \sigma_{2}\right](m),\left[V_{1}, V_{2}\right](p)\right) \quad m=\mu(p) .
$$


which is an element of $\mathcal{L}^{V} P$, since $\left[\sigma_{1}, \sigma_{2}\right](m) \in V_{m}$.

In what follows we further specialise to the case where the bundle $P$ is just $E$. This is the space where Lagrangian-type systems can be defined, as will be shown below. The main observation to make in this case is that we have a canonical map which allows a kind of splitting.

We recall that a splitting of the sequence $0 \longrightarrow V \stackrel{i}{\longrightarrow} \tilde{A} \stackrel{j}{\longrightarrow} \mathbb{R} \longrightarrow 0$ is simply a choice of a point of $A$. (Indeed, if we have a splitting $h$ and we put $h(1)=z$ then $j(z)=j(h(1))=1$, so that $z=i(a)$ for some $a \in A)$. Therefore, once we have fixed a point $a \in A$ we have two complementary projectors, a 'horizontal' one mapping $z$ to $j(z) i(a)$ and a 'vertical' one $\vartheta_{a}: \tilde{A} \rightarrow \tilde{A}$ given by $\vartheta_{a}(z)=z-j(z) i(a)$. Notice that the image of $\vartheta_{a}$ is in $V$, so it can be considered as a map form $\tilde{E}$ to $V$. (Indeed $j\left(\vartheta_{a}(z)\right)=j(z-j(z) i(a))=j(z)-j(z) \cdot 1=0$, so that $\vartheta_{a}(z)$ is in the image of $\boldsymbol{i}$.)

In the case of an affine bundle, we therefore have a map $\vartheta_{a}: \tilde{E}_{m} \rightarrow \tilde{E}_{m}$ for every $a \in E_{m}$, and thus a map $\vartheta: \tau^{*} \tilde{E} \rightarrow \tau^{*} \tilde{E}$ given by

$$
\vartheta(a, z)=(a, z-j(z) i(a)) .
$$

This map is called the canonical map. As mentioned above, we can consider it as a map $\vartheta: \tau^{*} \tilde{E} \rightarrow \tau^{*} V$. In local coordinates, we have

$$
\vartheta=\left(e^{\alpha}-y^{\alpha} e^{0}\right) \otimes e_{\alpha} .
$$

An important concept for the study of dynamical systems on affine Lie algebroids is that of admissible elements. An element $Z \in \mathcal{L}^{\tilde{E}} E$ is said to be admissible if, roughly, it has the same projection under $\tau_{1}$ and $\tau_{2}$. More precisely, we set

$$
\operatorname{Adm}(E)=\left\{Z \in \mathcal{L}^{\tilde{E}} E \mid \tau_{2}(Z)=i\left(\tau_{1}(Z)\right)\right\} .
$$

An equivalent characterisation is the following: $Z$ is admissible if and only if it belongs to $\mathcal{J}^{E} E$ and $\vartheta$ vanishes on its projection to $\tau^{*} \tilde{E}$, which we will denote by $\tau_{12}(Z)$ (cf. 16]), hence

$$
\operatorname{Adm}(E)=\left\{Z \in \mathcal{J}^{E} E \mid \vartheta\left(\tau_{12}(Z)\right)=0\right\} .
$$

Indeed, if $Z$ is of the form $Z=\left(z, V_{a}\right)$, then the first condition means that $z$ is in the image of $i$ and the second then further specifies that $z=i(a)$.

By a contact 1-form we mean a 1-form $\theta$ on $\mathcal{L}^{\tilde{E}} E$ (i.e. a $C^{\infty}(E)$-linear map from sections of $\tau_{1}$ to $C^{\infty}(E)$ ), which vanishes on sections whose image lies in $\operatorname{Adm}(E)$. It follows from the characterisation of admissible elements that contact forms are locally spanned by

$$
\theta^{\alpha}=\mathcal{X}^{\alpha}-y^{\alpha} \mathcal{X}^{0}
$$

Any 1 -form $\theta$ on $\tilde{E}$ determines a contact 1 -form $\bar{\theta}$ by means of the canonical map:

$$
\langle\bar{\theta}, Z\rangle=\left\langle\theta, \vartheta\left(\tau_{12}(Z)\right)\right\rangle .
$$


In coordinates, if $\theta$ is of the form $\theta=\theta_{0} e^{0}+\theta_{\alpha} e^{\alpha}$ then

$$
\bar{\theta}=\theta_{\alpha}\left(\mathcal{X}^{\alpha}-y^{\alpha} \mathcal{X}^{0}\right) .
$$

Notice that the elements of the basis $\left\{\theta^{\alpha}\right\}$ of contact 1-forms are of this type: $\theta^{\alpha}=\overline{e^{\alpha}}$. We further will need the affine function $\widehat{\theta} \in C^{\infty}(E)$ associated to $i^{*}(\theta)$. To be precise, there is of course a linear function on $\tilde{E}$ associated to $\theta$, but we will reserve the notation $\widehat{\theta}$ for its restriction to $E$, meaning that in coordinates:

$$
\widehat{\theta}=\theta_{0}+\theta_{\alpha} y^{\alpha} \text {. }
$$

With these definitions, we can split (the pullback of) a 1-form $\theta$ on $\tilde{E}$ as follows

$$
\tau_{2}^{*} \theta=\widehat{\theta} \mathcal{X}^{0}+\bar{\theta} .
$$

This decomposition is important for various calculations in the next section.

\section{Complete and vertical lifts}

The prolongation structure we have specialised to, with $F=\tilde{E}$ and $P=E$, is visualised in the following diagram.

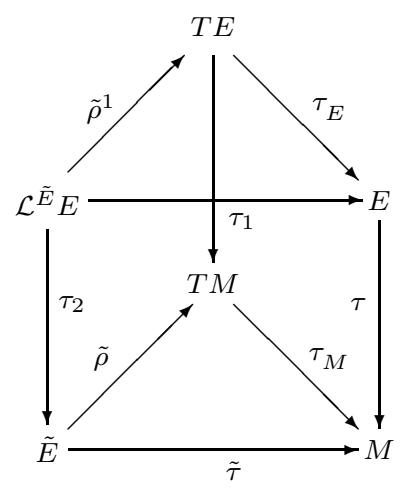

Given a section $\zeta$ of $\tilde{E}$ we can define two sections $\zeta^{C}$ and $\zeta^{V}$ of $\mathcal{L}^{\tilde{E}} E$ which are called the complete lift and vertical lift of $\zeta$, respectively.

The vertical lift is defined by the following sequence of natural constructions. Given a point $a \in E_{m}$ and a vector $\boldsymbol{v} \in V_{m}$, we define the vector $v_{a}^{V} \in T_{a} E$ by its action on functions $f \in C^{\infty}(E)$ :

$$
v_{a}^{V}(f)=\left.\frac{d}{d t} f(a+t v)\right|_{t=0} .
$$

Next, if $z \in \tilde{E}_{m}$ and $a \in E_{m}$, the vertical lift of $z$ to the point $a$ is defined as

$$
z_{a}^{V}=\left(\vartheta_{a}(z)\right)_{a}^{V} .
$$


For the special case that $z=\boldsymbol{i}(\boldsymbol{v})$, this is consistent with the preceding step: $z_{a}^{V}=\boldsymbol{v}_{a}^{V}$. Let now $\xi^{V}: \tau^{*} \tilde{E} \rightarrow \mathcal{L}^{\tilde{E}} E$ be the vertical lift map, determined by $\xi^{V}(a, z)=\left(0_{\tau(a)}, z_{a}^{V}\right)$. The final step in the construction now is obvious: if $\zeta$ is a section of $\tilde{E}$, we define the section $\zeta^{V}$ of $\mathcal{L}^{V} E$, called the vertical lift of $\zeta$, by putting

$$
\zeta^{V}(a)=\xi^{V}(a, \zeta(m)), \quad \text { with } a \in E \text { and } m=\tau(a) .
$$

In coordinates, if $\zeta=\zeta^{0} e_{0}+\zeta^{\alpha} e_{\alpha}$ then

$$
\zeta^{V}=\left(\zeta^{\alpha}-y^{\alpha} \zeta^{0}\right) \mathcal{V}_{\alpha}
$$

Much of the structure here discussed is encoded in the definition of a vertical endomorphism $S$ on $\mathcal{L}^{\tilde{E}} E$ (or sections of it), which goes as follows: $S=\xi^{V} \circ \vartheta \circ$ $\tau_{12}$. Explicitly, if $Z=\left(z, V_{a}\right)$, then $S\left(z, V_{a}\right)=\xi^{V}\left(\vartheta_{a}(z)\right)$. In coordinates, the type $(1,1)$ tensor field $S$ reads

$$
S=\left(\mathcal{X}^{\alpha}-y^{\alpha} \mathcal{X}^{0}\right) \otimes \mathcal{V}_{\alpha}
$$

The complete lift of a section $\zeta$ of $\tilde{E}$ is defined by the following two conditions which completely characterise it:

- $\zeta^{C}$ projects to $\zeta$, i.e. $\tau_{2} \circ \zeta^{C}=\zeta \circ \tau$, and

- $\zeta^{C}$ preserves the set of contact forms, that is, if $\theta$ on $\mathcal{L}^{\tilde{E}} E$ is a contact form then $d_{\zeta^{C}} \theta=\left[i_{\zeta^{C}}, d\right] \theta$ is contact.

In the case of the pullback of a 1 -form on $\tilde{E}$, making use of the decomposition as sum of a contact plus a non-contact form $\tau_{2}^{*} \theta=\widehat{\theta} \mathcal{X}^{0}+\bar{\theta}$, and taking into account that $\zeta^{C}$ projects onto $\zeta$ and that $\tau_{2}$ is a morphism of Lie algebroids, one can verify that

$$
\begin{aligned}
& d_{\zeta^{C}} \bar{\theta}=\overline{d_{\zeta} \theta}+\widehat{\theta} \overline{d_{\zeta} e^{0}}, \\
& d_{\zeta^{C}} \widehat{\theta}=\widehat{d_{\zeta} \theta}-\widehat{\theta} \widehat{d_{\zeta} e^{0}} .
\end{aligned}
$$

In fact, any of these two conditions is equivalent to the second condition in our definition of complete lift. is

The coordinate expression of the complete lift of the section $\zeta=\zeta^{0} e_{0}+\zeta^{\alpha} e_{\alpha}$

$$
\zeta^{C}=\zeta^{0} \mathcal{X}_{0}+\zeta^{\alpha} \mathcal{X}_{\alpha}+\left[\left(\dot{\zeta}^{\alpha}-y^{\alpha} \dot{\zeta}^{0}\right)+C_{\beta}^{\alpha}\left(\zeta^{\beta}-y^{\beta} \zeta^{0}\right)\right] \mathcal{V}_{\alpha},
$$

where $C_{\beta}^{\alpha}=C_{0 \beta}^{\alpha}+C_{\gamma \beta}^{\alpha} y^{\gamma}$ and, for a function $f \in C^{\infty}(M)$, the complete lift $\dot{f} \in C^{\infty}(E)$ is defined by $\dot{f}=\widehat{d f}$. The first two terms of $\zeta^{C}$ are determined by the projectability condition, whereas the third term can be obtained by applying the preceding formula to $\theta=e_{\alpha}$.

The vertical and complete lift satisfy the following properties

$$
\begin{array}{ll}
d_{\zeta^{v}} f=0 & d_{\zeta^{v}} \widehat{\theta}=i_{\zeta^{C}} \bar{\theta} \\
d_{\zeta^{C}} f=d_{\zeta} f & d_{\zeta^{C}} \widehat{\theta}=\widehat{d_{\zeta} \theta}-\widehat{\theta} \widehat{d_{\zeta^{c}}}
\end{array}
$$


for $f \in C^{\infty}(M)$ and $\theta$ a 1 -form on $\tilde{E}$. We prove only the third; if $\boldsymbol{v}=\vartheta_{a}(\zeta)$ then

$$
\begin{aligned}
d_{\zeta^{V}} \widehat{\theta}(a) & =\zeta(m)_{a}^{V} \widehat{\theta}=\left.\frac{d}{d t} \widehat{\theta}(a+t \boldsymbol{v})\right|_{t=0}=\left.\frac{d}{d t}\left(\widehat{\theta}(a)+t\left\langle\boldsymbol{\theta}_{m}, \boldsymbol{v}\right\rangle\right)\right|_{t=0} \\
& =\left\langle\boldsymbol{\theta}_{m}, \boldsymbol{v}\right\rangle=\left\langle\boldsymbol{\theta}_{m}, \vartheta_{a}(\zeta)\right\rangle=\left(i_{\zeta^{C}} \bar{\theta}\right)(a) .
\end{aligned}
$$

Also, it follows form the definition of the vertical endomorphism that

$$
S\left(\zeta^{C}\right)=\zeta^{V} \quad S\left(\zeta^{V}\right)=0 .
$$

Using the above equations it is a matter of a routine calculation to prove the following commutation relations.

$$
\begin{aligned}
& {\left[\zeta_{1}^{C}, \zeta_{2}^{C}\right]=\left[\zeta_{1}, \zeta_{2}\right]^{C}} \\
& {\left[\zeta_{1}^{C}, \zeta_{2}^{V}\right]=\left[\zeta_{1}, \zeta_{2}\right]^{V}+\left\langle\zeta_{1}, e^{0}\right\rangle \zeta_{2}^{V}} \\
& {\left[\zeta_{1}^{V}, \zeta_{2}^{V}\right]=\left\langle\zeta_{1}, e^{0}\right\rangle \zeta_{2}^{V}-\left\langle\zeta_{2}, e^{0}\right\rangle \zeta_{1}^{V}}
\end{aligned}
$$

The above definitions and relations are greatly simplified if we restrict to sections of the associated vector bundle $V$. Indeed, if $\boldsymbol{\sigma}=\sigma^{\alpha} e_{\alpha}$ is a section of $V$, then the complete and vertical lifts of $\boldsymbol{\sigma}$ have the coordinate expressions

$$
\boldsymbol{\sigma}^{C}=\sigma^{\alpha} \mathcal{X}_{\alpha}+\left(\dot{\sigma}^{\alpha}+C_{\beta}^{\alpha} \sigma^{\beta}\right) \mathcal{V}_{\alpha} \quad \sigma^{V}=\sigma^{\alpha} \mathcal{V}_{\alpha},
$$

and the action of the complete lift over linear functions and contact forms is given by

$$
d_{\sigma^{C}} \bar{\theta}=\overline{d_{\boldsymbol{\sigma}} \theta} \quad d_{\zeta^{C}} \widehat{\theta}=\widehat{d_{\boldsymbol{\sigma}} \theta}
$$

since $d_{\boldsymbol{\sigma}} e^{0}=0$. Furthermore, the commutation relations are as in the usual vector Lie algebroid case:

$$
\left[\boldsymbol{\sigma}^{C}, \boldsymbol{\eta}^{C}\right]=[\boldsymbol{\sigma}, \boldsymbol{\eta}]^{C} \quad\left[\boldsymbol{\sigma}^{C}, \boldsymbol{\eta}^{V}\right]=[\boldsymbol{\sigma}, \boldsymbol{\eta}]^{V} \quad\left[\boldsymbol{\sigma}^{V}, \boldsymbol{\eta}^{V}\right]=0 .
$$

\section{Lagrangian-type systems on an affine alge- broid}

In this section, we first consider dynamical systems on $E$, which geometrically are defined in the way standard second-order differential equations on a tangent bundle or first-jet bundle are conceived, but do not necessarily correspond, locally, to second-order equations. As in [20] therefore, we will call them pseudoSODEs. We shall subsequently discuss a class of pseudo-SODEs, which come from a (constrained) variational problem and therefore are said to be of Lagrangian type.

A curve $\gamma: \mathbb{R} \rightarrow E$ is said to be admissible if

$$
\rho \circ \gamma=\dot{\gamma}_{M},
$$


where $\gamma_{M}=\tau \circ \gamma$ is the projected curve to the base. In coordinates, if $\gamma(t)=$ $\left(x^{i}(t), y^{\alpha}(t)\right)$ then $\gamma$ is admissible if

$$
\dot{x}^{i}(t)=\rho_{0}^{i}(x(t))+\rho_{\alpha}^{i}(x(t)) y^{\alpha}(t) .
$$

A curve is admissible if and only if its prolongation takes values in the set of admissible elements. Indeed, the prolongation of the curve $\gamma$ is the curve $\gamma^{c}(t)=(i(\gamma(t)), \dot{\gamma}(t))$, and this is an admissible element if and only if it is in $\mathcal{L}^{\tilde{E}} E$, that is $\rho(\gamma(t))=T \tau(\dot{\gamma})(t)=\dot{\gamma}_{M}(t)$.

Definition 4. A pseudo-SODE on $E$ is a section $\Gamma$ of $\operatorname{Adm}(E)$, that is, a section of $\mathcal{L}^{\tilde{E}} E$ which takes values in the set of admissible elements.

From this definition, it readily follows that $\left\langle\Gamma, \mathcal{X}^{0}\right\rangle=1$ and the integral curves of $\tilde{\rho}^{1}(\Gamma)$ are admissible curves. Conversely, any section $Z$ of $\mathcal{L}^{\tilde{E}} E$ such that the integral curves of $\tilde{\rho}^{1}(Z)$ are admissible is a pseudo-sodE. From the alternative characterisation of $\operatorname{Adm}(E)$ as the set of elements of $\mathcal{J}^{E} E$ which vanish under $\vartheta$, it follows that a section $\Gamma$ of $\mathcal{L}^{\tilde{E}} E$ is a pseudo-SODE if and only if $S(\Gamma)=0$ and $\left\langle\Gamma, \mathcal{X}^{0}\right\rangle=1$.

Locally, a pseudo-SODE $\Gamma$ is of the form

$$
\Gamma=\mathcal{X}_{0}+y^{\alpha} \mathcal{X}_{\alpha}+F^{\alpha} \mathcal{V}_{\alpha}
$$

and the vector field $\tilde{\rho}^{1}(\Gamma)$ is of the form

$$
\tilde{\rho}^{1}(\Gamma)=\left(\rho_{0}^{i}+\rho_{\alpha}^{i} y^{\alpha}\right) \frac{\partial}{\partial x^{i}}+F^{\alpha} \frac{\partial}{\partial y^{\alpha}} .
$$

Now, to define Lagrangian-type equations in a coordinate free way, we can (as in [16]) simply mimic the usual construction on a first-jet bundle. For a given function $L$ on $E$, we define the Cartan 1-form $\Theta_{L}$ on $\mathcal{L}^{\tilde{E}} E$ by

$$
\Theta_{L}=d L \circ S+L \mathcal{X}^{0}
$$

and the Cartan 2-form $\Omega_{L}$ by $\Omega_{L}=-d \Theta_{L}$. We say that a pseudo-SoDE $\Gamma$ is of Lagrangian type if

$$
i_{\Gamma} \Omega_{L}=0 .
$$

If the Lagrangian is regular (the 2 -form $\Omega_{L}$ has maximal rank at every point) then there are no other solutions than multiples of a pseudo-SODE, but in the singular case this is a condition to be imposed.

In coordinates, we get

$$
\Theta_{L}=\frac{\partial L}{\partial y^{\alpha}} \theta^{\alpha}+L \mathcal{X}^{0}
$$

and the expression of $\Omega_{L}$ is simplified by fixing an arbitrary pseudo-SODE $\Gamma_{0}=$ $\mathcal{X}_{0}+y^{\alpha} \mathcal{X}_{\alpha}+F_{0}^{\alpha} \mathcal{V}_{\alpha}$ and using the basis $\left\{\mathcal{X}^{0}, \theta^{\alpha}, \psi^{\alpha}=\mathcal{V}^{\alpha}-F_{0}^{\alpha} \mathcal{X}^{0}\right\}$ dual to 


$$
\begin{aligned}
\left\{\Gamma_{0}, \mathcal{X}_{\alpha}, \mathcal{V}_{\alpha}\right\} & \\
\Omega_{L} & =\left(d_{\Gamma_{0}}\left(\frac{\partial L}{\partial y^{\alpha}}\right)-\frac{\partial L}{\partial y^{\gamma}} C_{\alpha}^{\gamma}-\rho_{\alpha}^{i} \frac{\partial L}{\partial x^{i}}\right) \theta^{\alpha} \wedge \mathcal{X}^{0} \\
& +\frac{\partial^{2} L}{\partial y^{\alpha} \partial y^{\beta}} \theta^{\alpha} \wedge \psi^{\beta}+\frac{1}{2}\left(\rho_{\beta}^{i} \frac{\partial^{2} L}{\partial x^{i} \partial y^{\alpha}}-\rho_{\alpha}^{i} \frac{\partial^{2} L}{\partial x^{i} \partial y^{\beta}}+\frac{\partial L}{\partial y^{\gamma}} C_{\alpha \beta}^{\gamma}\right) \theta^{\alpha} \wedge \theta^{\beta} .
\end{aligned}
$$

Lagrangian-type pseudo-SODE-equations are of the form

$$
\begin{aligned}
\dot{x}^{i} & =\rho_{0}^{i}+\rho_{\alpha}^{i} y^{\alpha}, \\
\frac{d}{d t}\left(\frac{\partial L}{\partial y^{\alpha}}\right) & =\rho_{\alpha}^{i} \frac{\partial L}{\partial x^{i}}+C_{\alpha}^{\gamma} \frac{\partial L}{\partial y^{\gamma}} .
\end{aligned}
$$

It is interesting to verify that such equations also can be obtained from a geometric calculus of variations approach. We explain how this works without working out all the technical details. Given a function $L \in C^{\infty}(E)$ and two points $m_{0}$ and $m_{1}$ on $M$, consider the problem of determining the critical curves of the functional

$$
J(\gamma)=\int_{\gamma} L=\int_{t_{0}}^{t_{1}} L(\gamma(t)) d t
$$

defined on the set of admissible curves $\gamma:\left[t_{0}, t_{1}\right] \rightarrow E$, for which $\gamma_{M}$ in the base manifold has fixed endpoints $m_{0}$ and $m_{1}$. This is a constrained problem, since the curves we consider are restricted to be admissible, i.e. they have to satisfy the constraints $\dot{x}^{i}=\rho_{0}^{i}+\rho_{\alpha}^{i} y^{\alpha}$. We should therefore be more specific about the class of admissible variations we will allow; they will be generated by complete lifts of sections of $V$, as follows.

Let $\boldsymbol{\sigma}$ be a section of $V$ such that $\boldsymbol{\sigma}\left(m_{0}\right)=\boldsymbol{\sigma}\left(m_{1}\right)=0$. We consider the vector fields $X=\boldsymbol{\rho}(\boldsymbol{\sigma})$ and $Y=\boldsymbol{\rho}^{1}\left(\boldsymbol{\sigma}^{C}\right)$, and we denote their flows by $\psi_{s}$ and $\Psi_{s}$, respectively. It follows that $\psi_{s}\left(m_{0}\right)=m_{0}$ and $\psi_{s}\left(m_{1}\right)=m_{1}$. The family of curves $\chi(s, t)=\Psi_{s}(\gamma(t))$ is a 1-parameter family of admissible variations of $\gamma$ : that $\chi(s, t)$ projects onto $\chi_{M}(s, t)=\psi_{s}\left(\gamma_{M}(t)\right)$ is obvious; the fact that $\chi(s, t)$ is an admissible curve for every fixed $s$ requires more work and is left to the reader. At the endpoints $t_{0}$ and $t_{1}$, we have

$$
\chi\left(s, t_{i}\right)=\Psi_{s}\left(\gamma\left(t_{i}\right)\right)=\psi_{s}\left(m_{i}\right)=m_{i} .
$$

The infinitesimal variation fields we consider are of the form $Z=Y \circ \gamma$; their projection to $M$ is $W=X \circ \gamma_{M}$. Therefore, the variation of $L$ along $\chi(s, t)$ at $s=0$ is given by

$$
\frac{\partial(L \circ \chi)}{\partial s}(0, t)=Z(t)(L)=Y(L)(\gamma(t))=\rho^{1}\left(\boldsymbol{\sigma}^{C}\right)(L)(\gamma(t))=d_{\boldsymbol{\sigma}^{C}} L(\gamma(t)),
$$

from which it follows that

$$
\left.\frac{d}{d s} J\left(\chi_{s}\right)\right|_{s=0}=\int_{\gamma} d_{\sigma^{C}} L
$$


If $\boldsymbol{\sigma}$ is a section satisfying the conditions given above, then so is $f \boldsymbol{\sigma}$ for every function $f$ on $M$. Taking into account that $(f \boldsymbol{\sigma})^{C}=f \boldsymbol{\sigma}^{C}+\dot{f} \boldsymbol{\sigma}^{V}$, we have that

$$
\begin{aligned}
0 & =\int_{\gamma} d_{(f \boldsymbol{\sigma})^{C}} L \\
& =\int_{\gamma} f d_{\boldsymbol{\sigma}^{C}} L+\dot{f} d_{\boldsymbol{\sigma}^{V}} L \\
& =\left.f\left\langle d L, \boldsymbol{\sigma}^{V}\right\rangle\right|_{\gamma\left(t_{0}\right)} ^{\gamma\left(t_{1}\right)}+\int_{\gamma} f\left\{d_{\boldsymbol{\sigma}^{C}} L-d_{\Gamma}\left\langle d L \circ S, \boldsymbol{\sigma}^{C}\right\rangle\right\} \\
& =\int_{\gamma} f i_{\boldsymbol{\sigma}^{C}}\left\{d L-d_{\Gamma}(d L \circ S)\right\}
\end{aligned}
$$

whereby $\Gamma$ is the pseudo-SODE of which the extremals we are looking for will be solutions, and we have made use of the property that $\left[\sigma^{C}, \Gamma\right]$ is vertical, as one can easily verify in coordinates.

Since $f$ is arbitrary, the fundamental lemma of the calculus of variations implies that its coefficient must vanish along extremals $\gamma(t)$ and therefore also in an open neighbourhood on $E$. So the vanishing of the variation of $J$ is equivalent to

$$
i_{\sigma^{C}}\left(d L-d_{\Gamma}(d L \circ S)\right)=0 .
$$

One easily verifies that $d_{\Gamma}(d L \circ S)-d L$ is 'semi-basic', and since it vanishes on the complete lift of arbitrary sections $\boldsymbol{\sigma}$ of $V$, it follows that $d_{\Gamma}(d L \circ S)-d L=\lambda \mathcal{X}^{0}$. The value of $\lambda$ can be found by contraction with $\Gamma$ :

$\lambda=\left\langle d_{\Gamma}(d L \circ S)-d L, \Gamma\right\rangle=i_{\Gamma} d_{\Gamma}(d L \circ S)-d_{\Gamma} L=d_{\Gamma} i_{\Gamma}(d L \circ S)-d_{\Gamma} L=-d_{\Gamma} L$.

Thus the Euler-Lagrange equations can be written as $d_{\Gamma} \Theta_{L}=d L$, from which it follows, since $d i_{\Gamma} \Theta_{L}=d L$, that $i_{\Gamma} \Omega_{L}=0$.

To finish, we also outline briefly the relation to a Hamilton-type formulation. For that we consider the prolongation of the extended dual, i.e. $\mathcal{L}^{\tilde{E}} E^{\dagger}$. In this bundle we have a canonical 1-form $\theta$ (cf. 15] or 17]) given by

$$
\left\langle\theta_{0},\left(z, V_{\varphi}\right)\right\rangle=\langle z, \varphi\rangle \text {. }
$$

In a local basis $\left\{\mathcal{X}^{0}, \mathcal{X}^{\alpha}, \mathcal{P}^{0}, \mathcal{P}^{\alpha}\right\}$ of $\mathcal{L}^{\tilde{E}} E^{\dagger}$ induced by a frame on $E$, the 1 -form $\theta_{0}$ reads

$$
\theta_{0}=\mu_{0} \mathcal{X}^{0}+\mu_{\alpha} \mathcal{X}^{\alpha}
$$

The canonical symplectic form $\omega_{0}$ is the 2 -form $\omega_{0}=-d \theta_{0}$, which in coordinates has the expression

$$
\omega_{0}=\mathcal{X}^{0} \wedge \mathcal{P}_{0}+\mathcal{X}^{\alpha} \wedge \mathcal{P}_{\alpha}+\mu_{\gamma} C_{0 \beta}^{\gamma} \mathcal{X}^{0} \wedge \mathcal{X}^{\beta}+\frac{1}{2} \mu_{\gamma} C_{\alpha \beta}^{\gamma} \mathcal{X}^{\alpha} \wedge \mathcal{X}^{\beta}
$$

The Lagrangian defines a map $\mathcal{F}_{L}$ from $E$ to $E^{\dagger}$, the Legendre transformation, defined as follows. The element $\mathcal{F}_{L}(a) \in E^{\dagger}$ is the affine approximation 
(first order Taylor polynomial) of $L$ at $a$. In other words, if $v$ is the vector such that $b=a+\boldsymbol{v}$ and $g$ is the function $g(t)=L(a+t \boldsymbol{v})$ then

$$
\mathcal{F}_{L}(a)(b)=g(0)+g^{\prime}(0)
$$

In coordinates

$$
\mathcal{F}_{L}\left(x^{i}, y^{\alpha}\right)=\left(x^{i}, L-\frac{\partial L}{\partial y^{\alpha}} y^{\alpha}, \frac{\partial L}{\partial y^{\alpha}}\right)
$$

Then we have, as in the standard theory, that the Cartan forms are the the pullback of the canonical forms by the prolongation of the Legendre transformation:

$$
\left(\mathcal{L F}_{L}\right)^{*} \theta_{0}=\Theta_{L} \quad \text { and } \quad\left(\mathcal{L F}_{L}\right)^{*} \omega_{0}=\Omega_{L}
$$

where $\mathcal{L} \mathcal{F}_{L}: \mathcal{L}^{\tilde{E}} E \rightarrow \mathcal{L}^{\tilde{E}} E^{\dagger}$ is the map

$$
\mathcal{L F}_{L}(z, V)=\left(z, T \mathcal{F}_{L}(V)\right)
$$

\section{Final comments}

The case we studied in $[20$ is when $M$ is fibred over the real line $\pi: M \rightarrow \mathbb{R}$ and the image of the anchor map $\rho$ belongs to $i\left(J^{1} M\right) \subset T M$. As said before, the motivation to investigate this particular case comes from potentially interesting applications of a time-dependent generalisation of the by now classical 'Lagrangian systems' on (vector) Lie algebroids (cf. [21]). Needless to say, we should be able to recover this case simply within the present more general setup. Essentially, in the case of the extra fibration, we have coordinates $\left(t, x^{i}\right)$, and we can think of $t$ as being the zeroth coordinate. Then, we have $\rho_{0}^{0}=1$ and $\rho_{i}^{0}=0$, so that

$$
\rho\left(e_{0}+y^{\alpha} e_{\alpha}\right)=\frac{\partial}{\partial t}+\left(\rho_{0}^{i}+\rho_{\alpha}^{i} y^{\alpha}\right) \frac{\partial}{\partial x^{i}}
$$

An interesting feature is that in this case we have $e^{0}=d t$, so that $e^{0}$ is not only closed but also exact. In fact, as argued in Section 6, this is the condition for a Lie algebroid structure on an affine bundle to have a 1-jet-valued anchor. There are corresponding changes, for example in the formula for the exterior derivative of a function and in the fundamental Poisson brackets on $E^{\dagger}$. We refer to 20] for this.

For the sake of clarity, however, it is useful to point out a rather subtle difference between the first and the present approach. In [20], we also discussed the notion of prolongation. Compared to the diagram we have here, in Section 9, the difference is that the bottom line was $E \rightarrow M$ rather than $\tilde{E} \rightarrow M$ and the prolonged bundle accordingly was an affine bundle rather than the vector bundle $\mathcal{L}^{\tilde{E}} E \rightarrow E$ we have here. In fact, for the purpose of defining (geometrically) time-dependent systems of Lagrangian type, one can construct all the necessary tools also on the affine prolongation of [20]. However, we wish to discuss in a forthcoming paper aspects such as the non-linear and linear connections which 
are naturally associated to a pseudo-SODE on an affine bundle $E$, and for that purpose, even specifically for the time-dependent framework, it turns out to be much more appropriate to use the prolongation structure of the diagram of Section 9 .

\section{References}

[1] Cannas da Silva A and Weinstein A, Geometric models for noncommutative algebras, Berkeley Mathematics Lecture Notes 10, American Mathematical Society, (1999).

[2] Cariñena JF, Ibort LA, Marmo G and Perelomov A, On the Geometry of Lie algebras and Poisson tensors, J. Phys. A: Math. Gen. 27 (1994) 7425-49.

[3] Courant T, Tangent Lie algebroids, J. Phys. A 27 (1994) 4527-4536.

[4] Crampin M, Jet bundle techniques in analytical mechanics, Quaderni del Consiglio Nazionale delle Ricerche, N. 47 (1995).

[5] Fernandes RL, Lie algebroids, holonomy and characteristic classes, preprint (2000), math.DG/0007132, Adv. Math., to appear.

[6] Grabowski J and Urbanski P, Lie algebroids and Poisson-Nijenhuis structures, Rep. Math. Phys. 40 (1997) 195-208.

[7] Grabowski J, Grabowska K and Urbanski P, Lie brackets on affine bundles, preprint (2002), math.DG/0203112

[8] Higgins PJ and Mackenzie K, Algebraic constructions in the category of Lie algebroids, J. of Algebra 129 (1990) 194-230.

[9] Iglesias D and Marrero JC, Some linear Jacobi structures on vector bundles, C. R. Acad. Sci. Paris Sr. I Math. 331 (2000) 125-130.

[10] Iglesias D and Marrero JC, Generalized Lie bi-algebroids and Jacobi structures, J. Geom. Phys. 40 (2001) 176-199.

[11] Libermann P, Lie algebroids and Mechanics, Arch. Math. (Brno) 32 (1996) 147-162.

[12] Mackenzie K, Lie groupoids and Lie algebroids in differential geometry, London Math. Soc. Lect. Note Series 124 (Cambridge Univ. Press) (1987).

[13] Mackenzie K, Lie algebroids and Lie pseudoalgebras, Bull. London Math. Soc. 27 (1995) 97-147.

[14] Mangiarotti L and Sardanashvily G, Connections in classical and quantum field theory, World Scientific Publishing (River Edge, NJ) (2000). 
[15] Martínez E, Geometric Formulation of Mechanics on Lie algebroids, In Procs. of the VIII Fall Workshop on Geometry and Physics, Medina del Campo 1999. Publicaciones de las RSME (2001).

[16] Martínez E, Lagrangian Mechanics on Lie algebroids, Acta Appl. Math. 67 (2001) 295-320.

[17] Martínez E, Hamiltonian Mechanics on Lie algebroids, preprint 2000

[18] Popescu M and Popescu P, Geometric objects defined by almost Lie structures, In Lie Algebroids, Banach Center Publications, 54 (2001) 217233.

[19] Sarlet W, Mestdag T and Martínez E, Lagrangian equations on affine Lie algebroids, In: Differential Geometry and its Applications, Proc. 8th Int. Conf. (Opava 2001), D. Krupka et al (Eds.), to appear.

[20] Sarlet W, Mestdag T and Martínez E, Lie algebroid structures on a class of affine bundles, preprint (2001), math.DG/0201264

[21] Weinstein A, Lagrangian Mechanics and groupoids, In: Mechanics day (Waterloo, ON, 1992), Fields Institute Communications 7, American Mathematical Society (1996) 207-231. 